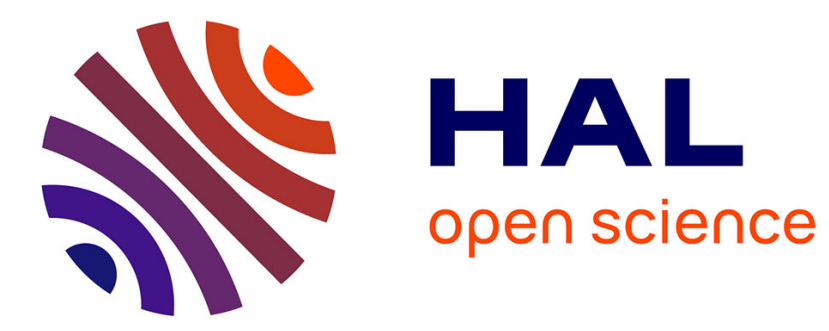

\title{
Trajectories of Attentional Development: An Exploration with the Master Activation Map Model
}

George A. Michael, Bernard Lété, Stéphanie Ducrot

\section{To cite this version:}

George A. Michael, Bernard Lété, Stéphanie Ducrot. Trajectories of Attentional Development: An Exploration with the Master Activation Map Model. Developmental Psychology, 2013, 49-4, pp.615631. 10.1037/a0028410. hal-01486674

\section{HAL Id: hal-01486674 https://hal.science/hal-01486674}

Submitted on 3 Feb 2022

HAL is a multi-disciplinary open access archive for the deposit and dissemination of scientific research documents, whether they are published or not. The documents may come from teaching and research institutions in France or abroad, or from public or private research centers.
L'archive ouverte pluridisciplinaire HAL, est destinée au dépôt et à la diffusion de documents scientifiques de niveau recherche, publiés ou non, émanant des établissements d'enseignement et de recherche français ou étrangers, des laboratoires publics ou privés. 
Trajectories of Attentional Development:

An Exploration with the Master Activation Map Model

George A. Michael

Laboratoire EMC \& University Lyon 2, Lyon, France

Bernard Lété

Laboratoire EMC \& University Lyon 2, Lyon, France

Stéphanie Ducrot

LPL-CNRS \& Université de Provence, France

\section{Correspondance}

George A. Michael

Université Lyon 2

Laboratoire d'Etude des Mécanismes Cognitifs

Département de Psychologie Cognitive \& Neuropsychologie

5, Avenue Pierre Mendès-France

69676 Bron Cedex

France

Tel : + (33).4.78.77.30.54.

Fax : + (33).4.78.77.43.51.

E-mail : George.Michael@univ-lyon2.fr 


\section{Abstract}

The developmental trajectories of several attention components, such as orienting, inhibition, and the guidance of selection by relevance (i.e., advance knowledge relevant to the task) were investigated in 498 participants (ages 7, 8, 9, 10, 11 and 20). The paradigm was based on Michael and al.'s (2006) Master Activation Map model and consisted of three visual search tasks presented in an intra-subject latin-square design and differing in terms of the probability with which a salient signal was associated with the target or a distractor. The results suggest that, whilst computations of salience were already proficient at age 7 , and the use of advance knowledge was efficient throughout childhood, albeit without reaching adult levels, the integration of salience and relevance reached its asymptotic level at age 8 . Whilst moving and engaging attention was proficient at age 7 , disengaging attention started to improve at age 9, reaching its adult level at age 11. As regards inhibition of salient distractors, we found no developmental pattern before adulthood, regardless of whether advance knowledge was available about the distractor or not, although all participants were able to use such knowledge to reduce overall interference. Finally, some results suggest that the control of resources for strengthening inhibition becomes efficient between ages 9 and 10. The developmental trajectories were compared with the existing literature and discussed.

Keywords: Attentional capture, visual search, salience, relevance, inhibition, Master Activation Map, children, development 


\section{Introduction}

How does attention develop towards the age point of highest efficiency? Several attention models have been proposed in the past, some of which, eg. the spot-light model, the zoom-lens model, or the feature integration theory (Posner, 1980; Eriksen \& St James, 1986; Treisman \& Gelade, 1980), have become powerful tools used world-wide for understanding attention-related phenomena and their development. More recently, more biologically plausible cognitive models have been proposed (Desimone \& Duncan, 1995; LaBerge, 1995; Michael et al., 2006), with greater account taken of interactions between bottom-up and top-down information flows. Constructed on the basis of the hybrid Master Activation Map model (MAMm; Michael et al., 2006) because of its complex structure encompassing interactions between bottom-up and top-down flows and its capacity to explain a wide range of attentional phenomena, the present series of experiments has multiple aims, namely to: (i) unravel how attention develops with age until reaching its adult level; (ii) use a complete and more complex model than past models, which takes account of bottom-up and top-down processing and how they interact; (iii) use simple experimental procedures combining modified aspects of known paradigms (e.g., cueing and visual search) with particular attention paid to a regular manipulation of instructions; (iv) extract a relatively large number of attention indices based on the same participants so that the final picture is as complete as possible; $(v)$ set up a larger number of groups of children so that smoother developmental patterns can be studied; (vi) set up relatively large samples of participants so that data are not contaminated with major fluctuations in chronometric performance; and, finally, (vii) inspect data for clues of independence or unilateral interdependence of computations and processes not yet postulated by the MAMm. 


\section{Relevant Developmental Patterns of Orienting and Search}

Visual selection of a single location in space can occur with (overt) or without (covert) the alignment of sensory receptors, i.e., without foveation. This is known as the orienting of attention (Posner, 1980) and is sought to involve basic operations such as disengaging attention from its current spatial locus, tagging this locus as explored (i.e., inhibition of return or loR; Posner \& Cohen, 1984; Klein, 1988), moving it away, and engaging attention on a new location. Everyday situations involve more complex behaviors than single location orienting, such as searching, which in turn involve hundreds of disengage-moveengage series. Orienting and searching involve many of the same processes and can be triggered involuntarily by visually salient signals or controlled voluntarily via ongoing goals and task demands. Even though involuntary and controlled components can be studied independently in laboratory situations, they usually combine to determine attentive behavior in everyday situations. In this paper, we present a selective overview of the findings relevant to the present study.

\section{Orienting to events without incentive}

As long as there is no particular incentive to orient to and search for a target (i.e. no particular motivation to explore one location in preference to another), the findings reported in the literature are rather unequivocal and consistent. Sudden peripheral changes and single items in an otherwise empty visual field, and items that differ from their otherwise homogenous neighbors in terms of their basic visual features and dimensions (Duncan \& Humphreys, 1989) can capture attention and trigger orienting responses towards their location. It has been suggested that age has seemingly little influence over orienting to a single location (Enns \& Cameron, 1987). Using a paradigm where participants were required to respond to a target preceded by a correct (valid) or misleading (invalid) peripheral cue that does not favor the development of spatial 
incentives, Enns and Brodeur (1989) found that 6 and 8 year-olds exhibit orienting patterns similar to those observed in adults. Trick and Enns (1998) reported such findings in a visual search task where participants had to detect a target presented alone or intermixed with a varying number of neighboring items. When the target was alone and when it was salient compared to its neighbors, detection of it was as efficient at age 6 as at 22. Other studies reported similar results (Hommel et al., 2004; Donnelly et al., 2007). There are also ubiquitous findings suggesting that pre-school children (under 5) can orient attention even in response to central symbolic cues that are not predictive (Ristic, Friesen \& Kingstone, 2002; Tipples, 2002; Ristic \& Kingstone, 2009). Overall, the available data suggest that moving attention overtly or covertly towards single locations, and locations exhibiting salience, and engaging at the location of sudden changes, are operations that are already functional and reach adult efficiency levels as early as 5-6 years of age.

However, there are other elementary operations of orienting which seem to change with age. For instance, if attention has just been engaged at a location not containing the target, it has to disengage and reorient elsewhere (Posner, 1980). Significant effects in response to misleading sudden spatial cues have been reported in 5 and 6 year-olds (Akhtar \& Enns, 1989; Enns \& Brodeur, 1989; Brodeur \& Boden, 2000), and these effects diminish with age. As well as being a sign that young children move attention towards those locations in response to cues, this also suggests that operations to disengage attention from its current locus are not fully functional. Steeper search slopes (i.e., time to process a single item; Ruskin \& Kaye, 1990; Trick \& Enns, 1998; Donnelly et al., 2007) in difficult visual search tasks where participants have no incentive to orient towards any particular item of the display, are concordant and contribute indirect findings that tap the disengage operation. Since moving and engaging are likely to be functional as early as 6 years of age (Enns \& Brodeur, 1989), the operation responsible for these developmental 
effects is most probably disengagement (Brodeur \& Boden, 2000). What is less clear, however, is the age at which disengagement reaches adult efficiency levels. For instance, Enns and Cameron (1987) reported that the age at which these levels are reached is as low as 7, while Enns and Brodeur (1989) and Brodeur and Boden (2000) found that, at 8 , efficiency is not yet at its adult level, and Akhtar and Enns (1989) found a similar lack of efficiency up to the age of 9 , with similar findings reported by Donnelly and colleagues (2007) for the performance of 9 to10 year-olds. There is clearly some evidence that disengagement develops through childhood, but no consensus exists on the age at which it is most efficient, as reflected through either spatial cueing paradigms or demanding visual search.

\section{Orienting to events with incentives}

The rise of incentives (i.e. motivation to explore one location in preference to another) can be based on the voluntary use of highly predictive symbolic central cues (Posner, 1980) or the use of highly predictive peripheral ones. The literature is very poor as regards the development of purely voluntary orienting to single locations, but there is evidence of developmental patterns. For instance, Ristic and Kingstone (2009) demonstrated that 5 year-olds have difficulty orienting in a purely voluntary way whilst others (Goldberg et al., 2001; Goldman et al., 2005; Wainwright \& Bryson, 2005) reported that 6 to 10 year-olds were as efficient as adults in orienting attention towards the cued location in response to highly predictive central cues. However, the findings reported as regards highly predictive peripheral cues are inconsistent. For instance, Enns and Brodeur (1989) found that children did not orient as efficiently as adults in response to $80 \%$ predictive peripheral cues, whilst Wainwright and Bryson (2002) found no difference between 6 year-old children and adults under the same conditions. Brodeur and Boden (2000) and Gupta and colleagues (2009) also reported no age effect for $67 \%$ and $100 \%$ predictive peripheral 
cues, respectively. These inconsistencies may be due, however, to individual differences in coordinating bottom-up and top-down operations when processing predictability associated with peripheral signals (larocci et al., 2009; Ristic \& Kingstone, 2009). Peripheral cues and salient items can acquire a particular status when they signal the most probable targets in that they also involve top-down, controlled processes based on relevance. It is suggested that integration of salience and relevance is not well developed in children (Ristic \& Kingstone, 2009).

\section{Relevant Developmental Patterns of Active Inhibition}

Active inhibition is the second major process involved in attentional selection. It is conceptualized as a general cognitive process (the fragmentation of which is possible) involved in all tasks requiring active control of, and resistance to interference, filtering, and avoidance and blocking of inappropriate and/or perseverative responses (Wager et al., 1995; Aron et al., 2004; 2007; Xue et al., 2009). It thus comes into play at both perceptual and motor levels and is seemingly dependent on the integrity of the frontal lobes, especially the right inferior frontal gyrus, including the frontal operculum (Aron et al., 2003; 2004; Michael et al., 2001b; 2006; Xue et al., 2008). Susceptibility to interference is a major developmental dimension (Dempster, 1993), making the assessment of inhibition throughout childhood critical for understanding attention.

Despite efficient use of central cues to orient attention, 6 year-olds seem to have difficulty adjusting the size and density of their attentional focus (Wainwright \& Bryson, 2005), an issue closely related to active inhibitory control (LaBerge, 1983; Eriksen \& St. James, 1986; Facoetti \& Molteni, 2000; Van der Lubbe \& Keuss, 2001). This difficulty seems to continue even beyond the age of 10 in spite of the fact that orienting has already reached its level of highest efficiency (Goldberg et al., 2001). Most developmental studies 
used varying versions of the flanker interference paradigm (Eriksen \& Eriksen, 1974) to assess the efficiency of inhibitory processes, whether in terms of the resolving of competition or filtering (Enns \& Girgus, 1985; Enns \& Cameron, 1987; Akhtar \& Enns, 1989; Enns, 1993; Ridderinkhof \& Van der Moler, 1995; Goldberg et al., 2001; Davidson et al., 2006). Unfortunately, even though there is evidence of developmental patterns, they are rather inconsistent. For instance, several studies (Enns \& Girgus, 1985; Akhtar \& Enns, 1989; Enns \& Cameron, 1987 Experiment 1) reported that interference from taskirrelevant distractors reduced through childhood but had not achieved adult levels even by age 10 (Enns \& Girgus, 1985; Goldberg et al., 2001). Other studies showed no interference effect in children (Enns \& Akhtar, 1989) or even interference that increased with age (Enns \& Cameron, 1987 Experiment 2). Finally, inconsistent findings were also reported for the behavioral and electrocortical effects of interference (Ridderinkhof \& Van der Moler, 1995). The former revealed a developmental decrease even between 10-12 year-olds and adults, whilst the latter were either stable up until adulthood (as indexed through the P3 latency) or showed a developmental trend similar to the one observed in behavior (as indexed through the P3 latency peak). To the extent that P3 latency and P3 peak latency reflect, respectively, perceptual and motor processes, it was suggested that the locus of developmental change was the ability to resist interference at the motor level.

Evidence from paradigms involving inhibition of unwanted responses (e.g., Stroop, gono go, stop-signal and response alternation) also tends to demonstrate that inhibitory processes continue to develop until early adolescence (Comalli, Wapner \& Werner, 1962; Wise, Sutton \& Gibbons, 1975; Levin et al., 1991; Brocki \& Bohlin, 2004; Johnstone et al., 2007; Williams et al., 1999; Bedard et al., 2002; Carver et al., 2001), even though inconsistent developmental patterns have been reported (Oosterlaan \& Sergeant, 1996; Schachar \& Logan, 1990; Johnstone et al., 2007). The dominant picture is that active 
inhibition seems to reach mastery in early adolescence, around the ages of 10 to 12 even though some studies suggest that this might be task-dependent, with mastery being reached faster in some tasks than others (Davidson et al., 2006). Such a result might be associated to asynchronies in the maturation of distinct brain circuits involved in inhibition (Bunge, 2002). The precise pattern of development through childhood is thus unclear and precludes any specific hypotheses.

\section{Overview of the Present Study and Hypotheses}

Despite the number of developmental studies on attention that have targeted orienting and inhibition, it is actually quite difficult to glean an overall, fairly complete and coherent picture of developmental patterns, for several reasons. First, not all studies were based on cognitive attention models. Models offer a precise frame for interpreting data and by this virtue they may better guide the interpretation of data. When they did, the results were frequently inconsistent and sometimes contradictory. This could be attributed to methodological differences, sample sizes, the choice of behavioral indices, or a combination of all of these. Second, all previously cited studies produced a puzzled image of how attention develops. Our purpose is therefore clear, namely to acquire a better overall picture of the development of attention processes up to the point of adult-like efficiency by assessing several components of attention in a very large number of participants by means of simple tasks constructed on the basis of a single, integrative and solid theoretical background, the MAMm (Michael et al., 2006; 2007).

Let us now attempt to predict performance in a visual search task deriving from previous studies (Michael et al., 2001a; 2001b; 2006). We present a protocol consisting of 3 independent visual search tasks which differ in terms of the probability of a salient signal 
being a target or distractor. In the first task, when the salient item is present, it is the target in only $50 \%$ of trials, as opposed to $100 \%$ of trials in the second task, whilst in the third task the salient item is always a distractor. All three tasks involve a complementary condition in which no salient item is present. The number of items varies randomly but systematically. Participants are informed about these settings, and this is intended to manipulate their orienting/search incentives, as well as the degree to which inhibitory control is involved. These tasks are taken both separately and in combination, an investigation which allows for the targeting of distinct performance indices.

\section{Orienting to events without incentives}

If, without any incentive, participants are searching for a particular target, then the presence of a salient item in the search display is expected to capture attention (Yantis \& Jonides, 1984). Since salient items are seemingly processed correctly in youngsters, no particular developmental patterns are to be expected. It can be supposed that children would perform as well as adults, exhibiting therefore faster response times (RTs) than in a condition where no salient item is present. Search efficiency would not depend, for any age group, on the number of items present in the display. Analyses should thus target the

effect of a salient target on response speed and search efficiency, and such indices would provide information about the salience-based orienting of attention (and, therefore, the computations underlying salience; Koch \& Ullman, 1985; Theeuwes, 1991; Michael et al., 2006). More specifically, the speed of search for a salient target would not depend on the number of items in the display, and youngsters would be expected to search as well as adults.

The absence of a salient item in the visual field and the absence of any incentives would lead to varying random patterns of exploring the display, with visual search 
progressing slowly on the basis of the disengage-move-engage loop until the target is found. The result of course would be steep search slopes in both adults and children, mostly because of the demanding disengagement operation. However, because this is an operation which develops throughout childhood, search slopes can be expected to diminish with age. Another interesting index is thus search efficiency in conditions where no salient items are present in the display, as this would yield information about the development of the disengagement operation as part of the orienting process. The search for a target would depend on the number of items present in the display and would become more efficient with age. Unfortunately, the literature is quite inconsistent as regards the age at which developmental patterns start to be visible and at which they reach adult levels, such that precise predictions are difficult to make.

The combination of efficient orienting towards a salient item and less efficient orienting towards a non-salient target would result in a specific developmental pattern, in other words major benefits for youngsters and decremental benefits with age. Such a pattern would be attributable mostly to the less efficient search in the absence of a salient target.

\section{Orienting to events with incentives}

If a salient signal were assigned a high probability of being the target, then participants would be expected to develop incentives to orient towards its location. This could be done by coordinating salience-based (bottom-up) and relevance-based (top-down) computations (Michael et al., 2006; Fecteau \& Munoz, 2006; Ristic \& Kingstone, 2009). The literature is quite inconsistent as regards this aspect of attention, preventing us therefore from proposing any sound hypothesis. An informative index would be the effects of salient signals as a function of their probability of being the target. If children use incentives to orient attention towards salient signals, their RT would decrease as the salient signal's 
probability of being the target increased. Comparing a condition in which the salient item is the target in $50 \%$ of the trials with one in which it is the target in $100 \%$ of trials should reveal faster RTs in the latter. Even youngsters should exhibit shorter RTs if they used incentives.

\section{Active inhibition}

As mentioned before, the presence of a salient item in the display would trigger involuntary movements of attention towards its location. What is to be expected if this specific salient item proves to be a distractor? According to Posner's spotlight theory (Posner, 1980), attention would disengage from the location of that item and then move away once inhibition of return (hereafter loR) applied. Research in our laboratory (Michael et al., 2001b; 2006) showed that inhibition is also activated and maintained throughout the whole period during which participants are searching for the target among the remaining items. In situations where participants know in advance that the salient item is most probably a distractor, inhibitory control is activated even before the display appears and maintained until the target is found. Compared with a situation where no salient item is present (i.e., a simple subtraction between a baseline and a salient-distractor condition) it would reveal the cost of inhibitory control. It is expected that (a) this cost would be less in situations where participants benefit from advance knowledge about the nature of the salient item. Furthermore, (b) whatever this advance knowledge, no developmental pattern would be observed through childhood. That is, the cost magnitude would be similar for youngsters and older children. Another important index to investigate is therefore the effect of a salient distractor condition compared with a condition where no salient item is present, and this should be done as a function of the probability of the salient item being the distractor. RTs should be slower in situations where a salient item is a distractor compared to a baseline. And if the salient item is always to be a distractor, inhibition should be more 
active, thus reducing the slowing in relation to a baseline. This would yield information about active inhibition during search, and the use of probabilistic signals to do so.

\section{General Method}

Figure 2 summarizes the protocol used in the three experiments. The stimuli were created on the basis of the widespread definition of salience (Koch \& Ullman, 1985; Duncan \& Humphreys, 1989), i.e., what made an item salient was the addition of a surrounding circle the basic dimensions of which (here, size and shape) made it unique compared to the other items of the display. Participants had to search for a target (a square opened on the left or right) presented among physically similar distractors (squares open along the top or bottom). The target and the distractors were present in each trial. Participants had to indicate the location of the target's gap (left vs. right) as quickly and accurately as possible by pressing a pre-defined key on a button box. In some trials, a salient item - a circle - was added in the display and contained the target or a distractor. In the salient target condition, the circle surrounded the target; in the distractor condition, it surrounded a distractor; and in the baseline condition, there was no salient item. The three experiments were defined as a function of the probability of the presence of the salient item in the target and distractor conditions. In Experiment 1, the salient item was either relevant (target condition) or irrelevant (distractor condition) in 1/3 of the trials. In Experiment 2, the salient item was relevant (target condition) in 1/2 of the trials and there was no distractor condition. In Experiment 3, the salient item was irrelevant (distractor condition) in $1 / 2$ of the trials and there was no target condition.

\section{FIGURE 2 ABOUT HERE}




\section{Participants}

A total of 498 participants took part in the present study. They were divided into 6 age groups: 7 years $(N=74,38$ males, $M=6$ years 10 months, $S D=3$ months $), 8$ years ( $N=100,60$ males, $M=7$ years 8 months, $S D=3$ months), 9 years $(N=93,51$ males, $M=8$ years 8 months, $S D=3$ months $), 10$ years $(N=80,52$ males, $M=9$ years 8 months, $S D=3$ months $), 11$ years $(N=77,41$ males, $M=11$ years, $S D=6$ months $)$, and 20 years ( $N=74,56$ males, $M=20$ years 3 months, $S D=22$ months). Children were from five different classes of four elementary schools in Gap, a town in southern France. All participants were native French-speakers and had normal or corrected-to-normal vision.

\section{Intra-subject latin-square design}

Because several conclusions about the functioning of attention processes can be drawn from comparing the three proposed Experiments, it was necessary to avoid order effects. Consequently, each Experiment was divided into three equivalent blocks ( $a, b$ and c), and the 9 resulting blocks were presented in a complete latin-square order (i.e., Exp1a$2 a-3 a-2 b-3 b-1 b-3 c-1 c-2 c)$ to each subject. Instructions and the tested condition were manipulated between the blocks (see Figure 2). This intra-subject procedure also controlled for learning, motivation and fatigue across the three Experiments, rendering their interpretation more plausible and their direct comparison possible. At the beginning of each new block, instructions were presented by the computer visually and verbally, and the experimenter made sure that the participant understood what he/she was required to do during the task. Each subject completed a 10-trial training session containing samples of all tested conditions, followed by the experimental session. The whole session lasted approximately 25 minutes. 


\section{Stimuli and Apparatus}

The stimuli were white outlined squares $\left(37.37 \mathrm{~cd} / \mathrm{m}^{2}\right)$ presented on a black background $\left(0.034 \mathrm{~cd} / \mathrm{m}^{2}\right)$. At a viewing distance of $30 \mathrm{~cm}$, the angular size of each square was $0.5^{\circ} \times 0.5^{\circ}$. Each square had a gap and was rotated $0^{\circ}, 90^{\circ}, 180^{\circ}$ or $270^{\circ}$ clockwise. When the use of a salient item was necessary, the concerned item was placed inside an outlined white circle $(37.37 \mathrm{~cd} / \mathrm{m} 2)$ with a radius of $1^{\circ}$. The target's orientation was $90^{\circ}$ or $270^{\circ}$, whilst that of non-targets was $0^{\circ}$ or $180^{\circ}$. The stimuli appeared on the monitor of a

DELL Precision M2300 computer equipped with a $2.40 \mathrm{GHz}$ processor and NVIDIA Quadro FX 360M graphics card.

\section{General Procedure}

The stimuli were randomly distributed inside an imaginary $7.8^{\circ} \times 7.8^{\circ}$ square, and the search display remained visible until a response was given. The whole session started with 10 training trials showing all possible conditions. A trial started with the appearance of a central fixation dot displayed for $1000 \mathrm{~ms}$. Then, the search display was presented. Three conditions were manipulated throughout the study, each occurring with equal probability: (a) in the baseline condition, the target differed from its neighbors only in its gap orientation; (b) in the salient target condition, the target was placed in the center of the salient item (circle); (c) in the salient distractor condition, one of the irrelevant items was placed in the center of the salient item. The display size was either 6 or 10 items, target included. The tested condition and display size varied randomly from trial to trial, the only constraint being the same number of trials presented within a single block for each condition and each display size. Participants were asked to indicate the location of the target's gap (right or left) by pressing one of two pre-defined response buttons as quickly as possible. The next trial started $500 \mathrm{~ms}$ after a response had been given. RTs and errors were recorded by the computer. Ninety trials were presented (15 trials per condition per 
display size) in three identical blocks of 30 trials each. All three conditions were included in Experiment 1 (a total of 90 trials), only the baseline and the salient target conditions were included in Experiment 2 (a total of 60 trials), and only the baseline and the salient distractor conditions were included in Experiment 3 (a total of 60 trials; Figure 2). Participants were fully informed about these settings.

TABLE 1 ABOUT HERE

\section{Experiment 1: Salient Item 50\% Task Relevant}

Experiment 1 investigated visual search processes and orienting towards salient items in the absence of any particular incentive. Salient items were targets on $1 / 3$ of trials, distractors on $1 / 3$ of trials and absent on $1 / 3$ of trials.

\section{Results}

The number of errors (Table 2) and medians of correct RT (Table 1) underwent an analysis of variance (ANOVA) with the tested condition (baseline, salient target, salient distractor) and display size (6 items vs. 10 items) as within-participants factor, and age as the between-groups factor. The Newman-Keuls test was used to conduct post-hoc comparisons, and targeted differences were analyzed with bicaudal $t$-tests. RT analyses were carried out on medians rather than means because of the intra-subject and betweenparticipants variability in RT classically observed in tasks of visual search 
Errors: The overall number of errors was very small. There was a significant main effect of condition $(F(2,984)=37.5, p<.001)$ with less errors in the salient target condition $(.71)$ than the baseline $(.82 ; p<.017)$ and the salient distractor condition $(1.09 ; p$ $<.001)$. The baseline and the salient distractor condition also differed $(p<.001)$. The main effect of age also proved significant $(F(5,492)=8.27, p<.001)$, with the number of errors decreasing with age (7 years: 1.33 ; 8 years: $.93 ; 9$ years: $1.24 ; 10$ years: $.83 ; 11$ years: .76; adults: .14) but diminishing significantly only in adults (compared to children all ps < .002). No other effects were observed.

$R T$ : All main effects and all interactions reached significance (all $p s<.002$ ). We focus here on the most important results. In the main effect of condition $(F(2,984)=1247.9$, $p<.001)$, compared to the baseline (1584 ms), RTs were faster in the salient target condition (1099 ms; $p<.001)$ and slower in the salient distractor condition (1891 ms; $p<.001)$. Furthermore, the salient target and salient distractor conditions differed from each other $(p<.001)$. Analysis of the Condition $x$ Display Size interaction $(F(2,984)=120.2 ; p<.001)$ revealed a pattern similar to that described in the literature (e.g., Yantis \& Jonides, 1984; Michael et al., 2006), with no effect of display size in the salient target condition (6 items: $1090 \mathrm{~ms} ; 10$ items: $1108 \mathrm{~ms}$; slope: $5 \mathrm{~ms} / \mathrm{item} ; p>.25$ ), and an impressive display size effect in both the baseline (6 items: 1406 ms; 10 items: 1762 ms; slope: 89 ms/item; $p<.001$ ) and salient distractor conditions (6 items: 1740 ms; 10 items: $2042 \mathrm{~ms}$; slope: $76 \mathrm{~ms} /$ item; $p<.001)$. These first results suggest that salient items captured attention. When these items were targets, they accelerated performance and decreased search behavior. When they were distractors, they slowed performance considerably. All age groups exhibited these effects of attentional capture albeit at different 
degrees (Figure 3A), as suggested by the Age $x$ Condition interaction $(F(10,984)=15.1$, $p<.001)$. Post-hoc Newman-Keuls tests between successive age groups revealed that the effects of a salient target (i.e., baseline minus salient target RT) were greatest for 7 yearolds (effect: $728 \mathrm{~ms}$ ), decreased linearly (8 years: $628 \mathrm{~ms} ; p<.06 ; 9$ years: $501 \mathrm{~ms} ; p<$ $.02)$ up to age 10 (407 ms; $p>$. 19) where there was a temporary asymptote (11 years: 415 ms; $p>$.83), and then decreased again in adulthood (231 ms; $p<.002)$. Benefits from a salient target thus decreased with age, although this is most probably due to youngsters having more difficulty finding the target in the baseline condition (Figure 3B). The effect of a salient distractor compared to the baseline was unchanged for children irrespective of age, but reduced after the age of 11 (11 years $v s .20$ years $t(149)=3.1, p<.002$; Figure 3B).

\section{FIGURE 3 ABOUT HERE}

The significance of the Age $x$ Condition $x$ Display Size interaction $(F(10,984)=2.86$; $p<.002)$ suggested differences in search slopes in the three conditions were due to age, insofar as the slopes decreased with age in the baseline $(F(5,492)=3.87, p<.002)$ and the salient distractor condition $(F(5,492)=4.16, p<.001)$, but not in the salient target condition $(F(5,492)=1.34, p>.24)$.

The results of Experiment 1 highlighted a number of interesting points. From a developmental perspective, differences in the amplitude of effects were observed, namely decreasing effects of salient targets compared to baseline, and stable effects of salient distractors from ages 7 to 11 , with a reliable decrement thereafter. Baseline search slopes were found to decrease with age, in contrast to salient target slopes, which remained unchanged. The large reduction with age in the benefits of a salient target is attributable 
not to fast orienting towards salient targets, which would mean youngsters orient faster, but to a less developed ability to orient attention towards locations not exhibiting salience. If large benefits signified faster orienting, greater costs should also be found for youngsters in the presence of a salient distractor, but this was not the case. Alternatively, different incentives to solve the salient target condition could have been used with increasing age. Given the large number of items in a display, young children could not have tried to avoid capture by the salient item, as adults could have done. In such a case, being captured by a salient item that contains the target would actually enhance performance, leading to better performance than adults. Yet, if this were the case, then the same would happen when salient distractors were concerned and the effects of a salient distractor would therefore follow a similar developmental trajectory. Instead, we found developmental stability up to age 11. This alternative cannot thus be maintained. Overall, we can conclude that (a) moving and engaging attention at the location of a salient target is as efficient at 7 as it is at 20; (b) demanding search becomes more efficient with age, which can primarily be attributed to the development of disengagement of attention from its current locus (see below for a more detailed discussion of this point after some betweenexperiments analyses); (c) at least one aspect of active inhibition, namely inhibition of a salient item, the relevance of which is unknown and which has just attracted attention, develops quite late.

TABLE 2 ABOUT HERE

\section{Experiment 2: Salient Item 100\% Task Relevant}

Experiment 2 was designed to investigate visual search processes and orienting towards salient items in the presence of incentives. This time, the salient item was always 
the target (i.e., no salient distractor was present) and participants were informed about this setting. The fact that this item is always task-relevant helps develop incentives to orient attention voluntarily towards salient signals and, thus, use combined salience-relevance information to speed up processing (Michael et al., 2006). The information provided by this Experiment about the use of incentives is only useful when compared with Experiment 1 (see below), but a separate analysis is required before comparing the two Experiments.

\section{Results}

The number of errors (Table 2) and medians of correct RT (Table 1) underwent an analysis of variance (ANOVA) with the tested condition (baseline, salient target) and display size ( 6 items vs. 10 items) as within-participants factor, and age as the betweengroups factor. The Newman-Keuls test was used to conduct post-hoc comparisons, and targeted differences were analyzed with bicaudal $t$-tests.

Errors: The main effect of condition reached significance $(F(1,492)=16.5, p<.001)$ with less errors in the salient target condition (.66) than the baseline (.81). The main effect of age was significant $(F(5,492)=5.71, p<.001)$, with the number of errors decreasing with age (7 years: .99; 8 years: .80; 9 years: $1.0 ; 10$ years: .75; 11 years: .70; adults: .16) but diminishing significantly only in adults (compared to children all $p s<.002$ ). No other effects were observed.

$R T$ : The main effect of condition proved significant $(F(1,492)=170.5, p<.001)$ with faster RTs in the salient target condition (969 ms) than the baseline (1468 ms). The Condition x Display Size interaction was also significant $(F(1,492)=95.05, p<.001)$ 
revealing the expected pattern, ie. a major display size effect in the baseline (6 items: 1364 ms; 10 items: $1571 \mathrm{~ms}$; slope: $52 \mathrm{~ms} /$ item; $p<.001$ ) and a very shallow display size effect in the salient target condition baseline (6 items: 954 ms; 10 items: 984 ms; slope: 8 $\mathrm{ms} /$ item; $p<.019)$. Even though this effect was significant, the shallow search slope suggests the visual search was very efficient and effortless. Finally, the Age $x$ Condition interaction also reached significance $(F(5,492)=27.9, p<.001)$. All age groups exhibited faster RTs in the salient target condition than in the baseline conditions (see Figure 4), with these effects decreasing with age (see Figure 5B). The Age $x$ Condition $x$ Display Size interaction did not reach significance $(F<1)$. By contrast to Experiment 1 , search slopes exhibited no developmental effect, not even in the baseline condition.

\section{FIGURE 4 ABOUT HERE}

\section{Experiment 3: Salient Item 100\% Task Irrelevant}

Experiment 3 was designed to investigate active inhibition of salient items. This time, the salient item was always a distractor (i.e., no salient target was present) and participants were informed about this setting. The fact that this item is always taskirrelevant helps avoid voluntary orientation towards salient signals (Michael et al., 2001; 2006), and thus the development of incentives to inhibit. The information provided by this Experiment about the use of incentives is only useful in comparison to Experiment 1 (see below), but a separate analysis is required before comparing the two Experiments.

\section{Results}

The number of errors (Table 2) and medians of correct RT (Table 1) underwent an analysis of variance (ANOVA) with the tested condition (baseline, salient distractor) and display size ( 6 items vs. 10 items) as within-participants factor, and age as the between- 
groups factor. The Newman-Keuls test was used to conduct post-hoc comparisons, and targeted differences were analyzed with bicaudal $t$-tests.

Errors: The main effect of condition reached significance $(F(1,492)=66.9, p<.001)$ with less errors in the baseline (.74) than the salient distractor condition (1.04). The main effect of age was significant $(F(5,492)=6.85, p<.001)$, with the number of errors decreasing with age (7 years: $1.34 ; 8$ years: $1.03 ; 9$ years: $1.16 ; 10$ years: $.85 ; 11$ years: .80 ; adults: .16) but the decrease was significant only in adults (compared to children all ps $<.003)$. No other effects were observed.

$R T$ : The main effect of condition was revealed to be significant $(F(1,492)=518.3$, $p<.001$ ), with RTs slower in the salient distractor condition (1785 ms) than the baseline (1570 ms). All age groups exhibited this difference (see Figure 5A) but to differing degrees as testified by the Age $\times$ Condition which reached marginal significance $(F(5$, $492)=2.15, p<.059)$. Closer inspection showed that the effect of the salient distractor remained unchanged throughout childhood, decreasing only thereafter (see Figure 5B; 11 years vs. 20 years $t(149)=2.97 ; p<.003)$. The Age $\times$ Condition $x$ Display size interaction failed to reach significance $(F(5,492)=0.82, p>.53)$.

FIGURE 5 ABOUT HERE

\section{Between-Experiment Comparisons}

The advantage of using an intra-subject latin-square design is the possibility of carrying out additional between-task analyses. 


\section{Preparatory and Setting Effects on Baselines and Their Search Slopes}

LaBerge and colleagues (2000) used the terms preparatory attention and brief attention to distinguish attention generated prior to the target display from attention generated at the time of the target display. Both aspects are selective, but the duration of preparatory aspects is longer than that of attention evoked at the time of display and involves expectations that a particular item will occur. From this point of view, participants' performance in each condition of each of the three Experiments reveals how brief attention operates, whilst between-Experiment comparisons may reveal preparatory aspects of attention as a result of instructions. Within this scope, the most interesting comparison for assessing preparatory attention is a comparison of baselines. In all three Experiments, baseline conditions were strictly identical and there was nothing to differentiate them apart from instructions concerning other conditions (e.g., salient target and salient distractor). If, as LaBerge and colleagues (2000) suggested, expectations about particular items are adjusted according to the instructions given and somehow kept stable during the blocks of trials, then preparatory effects should be found. More specifically, in comparison to Experiment 1, where participants had no particular expectations about the salient item, RTs in the baseline condition should be faster in Experiment 2, where participants expected salience always signaled the target, and slower in Experiment 3 where they expected salience always signaled a distractor.

An analysis of variance (ANOVA) was carried out on baseline correct median RTs, with the experimental context (Exp1, Exp2, Exp3) and display size (6 items vs. 10 items) as within-participants factors, and age group as between-groups factor. The main effect of context reached significance $(F(2,984)=43.9, p<.001)$ due to faster RTs in Experiment 2 (1468 ms) compared to both Experiments 1 (1584 ms, $p<.001)$ and 3 (1570 ms, $p<.001)$, the difference between Experiments 1 and 3 being non significant $(p>.29)$. The 
Age $x$ Context interaction was marginally significant $(F(10,984)=1.81, p<.055$; Figure $6 \mathrm{~A})$ and was investigated further by comparing Experiments 2 and 3 with Experiment 1 with the help of paired bicaudal $t$-tests (Figure 6B).

\section{FIGURE 6 ABOUT HERE}

The analysis of this interaction revealed that all groups exhibited faster RTs in Experiment 2 than in Experiment 1 (all ps <.002). Although only adults differed from the other groups in that their preparatory effects were smaller, Figure 6B shows an overall tendency for this effect to decrease with age, principally after the age of 9 . On the other hand, the comparison between Experiments 1 and 3 revealed a slowing of RTs in Experiment 3 that decreased with age and became facilitatory at age 9, remaining stable thereafter. This facilitation was not significant for children (all ps $>.11$ ) but was significant in adults $(p<.012)$. Finally, the Age $x$ Display Size interaction was significant $(F(5,492)=$ $7.94, p<.001$; Figure $6 \mathrm{C})$, with the decrement of search slopes starting at the age of 9 and reaching an asymptotic level at 11 . The Context $x$ Display Size interaction was significant $(F(2,984)=17.07, p<.001)$, due to shallower search slopes in Experiment 2 (52 ms/item) than in both Experiments 1 (89 ms/item; $t(497)=6.33, p<.001)$ and $3(75$ ms/item; $t(497)=5.79, p<.001)$. The slope between Experiments 1 and $3(t(497)=7.17$, $p<.001$ ) also differed. In Experiment 3 participants knew in advance that the salient item was never a target. Even if this item captured their attention, they could have avoided exploring it in detail so that there was one less item to explore. Although context effects were found mainly in Experiment 2, a highly significant positive correlation was found between facilitatory (Exp1 minus Exp2) and inhibitory effects (Exp3 minus Exp1), revealing that these functions are interrelated $(r(496)=.45, p<.001)$. 


\section{Salient Item $50 \%$ vs. $100 \%$ Task-Relevant (Exp1 vs. Exp2)}

An ANOVA was conducted on correct median RTs obtained in the salient target condition, with the probability of the salient item being the target (50\% vs. $100 \%)$ and display size (6 vs. 10 items) as within-participants factors, and age group as the betweengroups factor. The main effect of probability was significant $(F(1,492)=262, p<.001)$, with RTs faster when the salient item was always the target (969 ms) than when it was in only half the trials (1099 ms). Only the Age x Probability interaction was significant $(F(5,492)=4.22, p<.001$; Figure $7 A)$, revealing that the use of probabilistic information associated with the relevance of a salient item was not the same across all ages. Post-hoc analyses showed that 7 year-olds benefited most from the use of such information (Figure 7B) since they differed from 8 year olds $(\mathrm{p}<0.042), 9$ year-olds $(p<0.012), 10$ year-olds $(p<0.007), 11$ year-olds $(p<0.00013)$ and adults $(p<0.02)$, but no significant change was found thereafter in spite of the smooth decrement (all differences between the other groups ps $>0.25)$.

\section{FIGURE 7 ABOUT HERE}

\section{Salient Item $50 \%$ vs. $100 \%$ Task-Irrelevant (Exp1 vs. Exp3)}

Previous research on patients suffering circumscribed lesions of the ventral areas of the frontal cortex (Michael et al., 2001b; 2006) suggested that the processes involved in processing salient task-irrelevant signals are the same, be they sometimes or always taskirrelevant. So what distinguishes these two situations is merely the use of probabilistic information about how frequently these signals occur. Comparing such conditions is therefore a way of assessing how such probabilistic information is used as a prerequisite for developing incentives to avoid salient signals by activating the inhibitory process. 
Experiments 1 and 3 both provided a fairly clear and harmonious picture of the efficient activation of inhibition: the changes it undergoes take place not in childhood but at some point after the age of 11 . However, is it the case that the development of incentives to activate inhibition follows the same pattern? To find out, we undertook a direct comparison of the salient distractor conditions of Experiment 1 and 3, insofar as the only difference between them was the contextual probability of the salient item being a distractor: 50\% in Experiment 1 and 100\% in Experiment 3.

An ANOVA was thus carried out on correct median RTs obtained in the salient distractor condition, with the probability of the salient item being a distractor $(50 \%$ vs. $100 \%$ ) and display size (6 vs. 10 items) as within-participants factors, and age group as the between-groups factor. The three-way Age x Probability x Display Size interaction reached significance $(F(5,492)=2.74, p<.019 ;$ Figure 8$)$, suggesting that the developmental pattern for the use of probabilistic information varied as a function of the number of items on the screen. All groups used probabilistic information equally well to reduce distraction when 6 items were displayed since the effect of probability $(50 \%$ minus $100 \%)$ was rather stable across ages $(F(5,492)=1.73 ; p>.012 ; 7$ years: $112 \mathrm{~ms} ; 8$ years: 103 ms; 9 years: 196 ms; 10 years: 50 ms; 11 years: 128 ms; adults: 148 ms; Figure $8 A$ ), but 7 and 8 year-olds were unable to benefit from the available advance knowledge when 10 items were displayed $(F(5,492)=2.78 ; p<.018 ; 7$ years: $5 \mathrm{~ms} ; 8$ years: -28 ms; 9 years: 86 ms; 10 years: 155 ms; 11 years: 179 ms; adults: 131 ms; Figure 8B).

\section{FIGURE 8 ABOUT HERE}

Increased probability that an item is not the target might strengthen inhibition (Michael et al., 2001; 2006). Even though the separate analyses of the distractor effects in 
Experiments 1 and 3 showed that the process of active inhibition per se undergoes no changes before the age of 11 , the combined analysis suggests that the use of probabilistic signals which may modulate the intensity of inhibition occurs at the age of 7 or 9 , depending apparently on the quantity of visual information that needs processing. The assumption is that top-down control of inhibition depends on resources (Watson \& Humphreys, 1997; Michael et al., 2006). When the display size is quite small, 7 and 8 year-olds reduce distraction, probably because sufficient resources are available for doing so. As the display size grows, however, more resources are needed for the demanding search, and the remaining resources are seemingly not sufficient to activate inhibition in such a way as to decrease efficiently the propensity for salient task-irrelevant signals to compete for selection. Based on what has been presented up until now, it can thus be concluded that (a) inhibition becomes efficient only after the age of 11 , (b) incentives to inhibit salient signals based on probabilistic information and context occur as early as 7 , provided that (c) enough cognitive resources are available for that to happen. 


\section{General Discussion}

The present study investigated several aspects of the development of attention processes throughout childhood. We achieved our aim by constructing simple experimental procedures that adapted and combined aspects of known paradigms and paid particular attention to the manipulation of instructions, and also by extracting a relatively large number of intra-individual attention indices so that the picture we obtained was as complete as possible. Lastly, to the best of our knowledge, our sample sizes are among the largest ever to have been included in a developmental study. With the goal of establishing smoother developmental patterns, we also opted for quite a large number of participant groups.

The paradigm involved visual search for a target. The manipulations we were interested in were the number of neighboring items, but most of all the presence or not of a different item in the display rendering one of the items salient. The probability of the salient item being the target or a distractor varied.

In this general discussion, the main results are described and compared with the existing literature. They are then briefly interpreted within the context of the MAMm. An attempt is made at the end to integrate these results within the MAMm.

\section{Orienting to events without incentives}

Available data suggest that the elementary attentional operations of moving and engaging, depending on subcortical brain structures such as the superior colliculus and pulvinar, respectively, (Posner \& Petersen, 1990), develop early and reach the levels of efficiency observed in adults as early as 5-6 years of age (Enns \& Brodeur, 1989; Trick \& Enns, 1998; Donnelly et al., 2007). These operations were investigated in Experiment 1, where participants had to find a target from among a varying number of neighbors. In 
some trials, an item was rendered salient and was only the target half of the time. In these conditions, there are only minimal incentives to orient towards the salient item (Yantis \& Jonides, 1984). We observed that search slopes for the target, when it was salient, were flattened in all age groups, suggesting that participants searched for the target efficiently. Indeed, RTs did not depend on the number of neighboring items, which suggests participants moved and engaged attention towards the target first. Furthermore, search slopes did not differ across the age groups, implying that moving attention and engaging it at the location of a salient target is as efficient at 7 as at 20 . This finding is in keeping with what was reported by Trick \& Enns (1998), namely that the efficiency of moving attention does not alter with age. So it can be assumed that, since salience-based orienting of attention is as efficient in children as in adults, the underlying computations (Koch \& Ullman, 1985; Theeuwes, 1991; Michael et al., 2006; Michael \& Gálvez-García, 2011), develop early and function similarly as we get older.

The invariable and flattened search slopes observed in the salient target condition of Experiment 1 contrasted with those obtained in the baseline condition, where no salient item was presented. A further clear and smooth developmental pattern was observed when the baselines of all three experiments were combined. Search slopes were quite steep and depended on the number of items in the display, revealing effortful search for the target. Slopes also decreased with age, which suggests that orienting from one item to another does not appear to be fully efficient in youngsters (Trick \& Enns, 1998), despite evidence showing that some orienting components develop quite early. Searching involves a series of disengage-move-engage operations until the target is found. And since, as suggested above, moving and engaging are likely to be functional in children as young as 6 (Enns \& Brodeur, 1989), this can be attributed principally to the development of attention disengagement from its current locus (Brodeur \& Boden, 2000). The combined search 
slopes of baselines in this study showed a slightly different pattern to those reported in the literature. In fact, no difference was observed up until age 8 , followed by a smooth but continuous developmental increment in search efficiency up to age 11 , and then no difference between the ages of 11 and 20 . This pattern is close to the one reported by Donnelly et al. (2007), i.e., an asymptotic level reached at age 9-10.

At this point we can propose that several operations are already functional at the age of 7: (a) detection and localization of signals in space, (b) the computations underlying the generation of salience (i.e., feature extraction and processing, comparison of each item to its neighbors for each feature, summation of the differences to derive a master salience activity), (c) moving of attention to the locus exhibiting salience and engaging it there. Conversely, the presence of a smooth developmental effect in trials where the target was difficult to distinguish from its neighbors strongly suggests that $(d)$ disengaging attention from its current locus does not start to develop before age 9 and reaches its high level of efficiency at 11 . It is quite clear that the orienting process does not develop as a single entity. Its component parts (moving, engaging and disengaging) start developing at different ages, and some of them take longer to develop than others.

\section{Orienting to Events with Incentives}

The baseline conditions of Experiments 1,2 and 3 were compared to investigate the use of incentives. These conditions were strictly identical, the only difference being the instruction given to participants prior to each block about the probability that the salient item could be the target. The first observation was a reliable overall acceleration of RTs in Experiment 2 compared to the other two Experiments, as well as shallower search slopes. In Experiment 2, participants were told that the salient item was always the target, an 
instruction which influenced chronometric performance even in trials where no such salient item was present. It appears that expectations about particular items are adjusted according to instructions and somehow remain stable during the blocks of trials (LaBerge et al., 2000). Their effects appear to be broad enough to influence all conditions, not only those specifically concerned. Thus, incentives accelerate processing speed and search efficiency.

The second group of observations was that all age groups exhibited this effect, that youngsters exhibited the strongest effect, and that the effect showed a very shallow decline with age (Figure 6B). The fact that the conditions were identical and no salient item was present precludes any interpretation of this result as reflecting more difficulty in searching for the target in youngsters. Instead, it is as if youngsters made more use of the instructions and improved their performance accordingly. This pattern is somewhat reminiscent of Akhtar and Enns (1989) who asserted that youngsters use all their resources to attend to the location of the most probable target without taking into account the possibility that the target could appear at other locations. However, such an assertion cannot fully explain our data since, in the baseline condition; the target's location could not have been known in advance. It may be possible, however, that all resources are used when participants know that, wherever it appears, a salient signal will always be the target, and thus there is no risk of making a mistake. The fact that preparing to process such a signal renders all resources available could account for both the general increment in RTs and some of the data from other paradigms (LaBerge et al., 2000), but the developmental pattern observed cannot be reconciled with this interpretation. The aforementioned results suggest that some components of orienting undergo little change with age, whilst others gain in efficiency. Here, however, it is clear that the effect of incentives is in stark contrast since it decreases with age. The suggestion is therefore that what is influenced and 
modulated by incentives (developed upon experimental context and instructions) is not the process of orienting per se, but probably the greater availability of resources. Youngsters might have difficulty managing resources and use them all in order to process the target (Akhtar \& Enns, 1989). Adults, on the other hand, may not use all the resources available to them to achieve that aim. Instead, they may just use those needed to allow them to progress quickly in their search without exhausting the whole stock. This differential use of resources may explain the decrement of task instruction effects with age.

Orienting could be affected in cases where specific information about the target is available. Orienting to events with incentives and its development were investigated by comparing the salient target condition between Experiments 1 and 2 . The salient item was the target in $50 \%$ of the trials in Experiment 1, but in $100 \%$ of trials in Experiment 2. Faster orienting towards the salient item was observed when the probability to contain the target was $100 \%$. This suggests of course that all age groups make good use of probabilities to integrate salience and relevance. An alternative interpretation would be that, instead of using probabilistic knowledge to orient attention, participants simply used different strategies in Experiments 1 and 2. Changing the probability that an item is the target may constitute a qualitative change in task demands. Developmental changes would, therefore, reflect use of different strategies through childhood. However, there are some methodological and empirical arguments that run counter to such an alternative. That salient target and baseline trials were intermixed and presented randomly prevents shifting and/or adjusting search strategies before the presentation of the display. Furthermore, if we admit that qualitative shifts in task demands occur, then some specific effects would be expected in the baseline condition of Experiment 2 when compared to the respective baselines of Experiment 1 (and Experiment 3, too). Indeed, if participants just searched for the circle, then the absence of this circle would have no effect (i.e., a quick-and-dirty 
rejection of the strategy followed by an item-to-item search for the target) rendering the baseline of Experiment 2 equivalent to the baselines of Experiments 1 and 3 . Alternatively, it would have a detrimental effect on performance compared to the baselines of Experiments 1 and 3 because what the participant sees in the baseline counters his/her preparatory set and strategy (i.e., find the circle), and this would slow down performance. None of these were found, this is why changes in search strategy are less probable than the use of probabilities.

In the past, several studies have attempted to assess the effects of cue predictability in Posner-like paradigms (Enns \& Brodeur, 1989; Brodeur \& Boden, 2000; larocci et al., 2009), but with conflicting results, due perhaps primarily to the comparison of orienting effects (i.e., the difference between situations where the cue was valid and those where it was not). For instance, Enns and Brodeur (2000) concluded that only adults were able to enhance the salience-based orienting effect with incentives under predictable conditions. The most interesting study was probably by larocci and colleagues (2009), who investigated orienting of attention without spatial precues, with peripheral precues only (i.e., salience-based orienting), with central precues only (i.e., relevance-based orienting), or with both peripheral and central precues (i.e., integrated orienting). They found that performance was always faster when participants received both peripheral and central precues rather than peripheral precues only. This effect was observed across all age groups, as in the present study, and its mere presence is consistent with the postulate of enhanced processing due to integration of salience and relevance (Michael et al., 2006; Fecteau \& Munoz, 2006). An initial conclusion is thus that all age groups make good use of probabilities to integrate salience and relevance. A further finding of our study, never observed before (larocci et al., 2009), was that 7 year-olds exhibited larger effects of probability. This is most probably due to the fact that they orient attention more slowly 
when the salient signal is less likely to be the target (i.e., 50\%), and thus the benefit of advance knowledge is greater. This interpretation is also consistent with the greater benefits observed in Experiment 1 between the baseline condition and the condition where the salient item was $50 \%$ task-relevant.

It is interesting to note that the trajectory of this effect was quite different to that observed when comparing the baselines of Experiments 1 and 2 (Figure 6). The previous pattern was relatively stable and underwent only minor changes after age 9 , whereas comparing the salient target conditions of these two experiments revealed an acute change after age 7 followed by a non-significant trend thereafter. These two results may appear to contradict each other, but the conditions they were triggered by actually involve a major difference, namely the presence of a salient item. Thus, the use of incentives generally and their use to orient attention probably involve different processes.

\section{Active Inhibition}

Active inhibition mostly applies to the location of a salient item that has already attracted attention and can still exert such a pull, and it will be maintained as long as the target is not found (Michael et al., 2006). Active inhibitory control was investigated by comparing situations where a salient distractor to be avoided was present in the search display with situations where no such item was present. In the salient distractor condition participants have to inhibit the salient item and continue their search while actively maintaining inhibition (Footnote 1). Irrespective of the probability of a salient signal being a distractor (Experiments 1 and 3), chronometric performance was slowed by its presence, but no developmental pattern at all was observed between the ages of 7 and 11 . This cost was reduced at age 20. In keeping with past findings (e.g., Enns \& Girgus, 1985; Levine et al., 1991; Goldberg et al., 2001; Bunge, 2002), this suggests inhibitory control per se is not 
mastered until quite late. Unfortunately, because there were no age groups between 11 and 20 in the present study, it is not possible to pinpoint the age at which inhibitory control starts to be achieved.

Is the inhibition strengthened by the probability that an item is not the target ? Even though the separate analyses of the distractor effects in Experiment 1 and 3 suggested that active inhibition per se does not really undergo changes before the age of 11 , a direct comparison of these two Experiments showed that the use of probabilistic signals may modulate the intensity of inhibition, and that this may develop through childhood. First, the overall distractor effect (independent of age) diminished as advance knowledge about the distracting nature of the salient item increased (50\%: $307 \mathrm{~ms} ; 100 \%: 215 \mathrm{~ms}$ ). Closer inspection of Figures $3 B$ and $5 B$ suggests strengthening of inhibition is visible as early as 7 and remains more or less the same thereafter. This first finding is highly interesting as it suggests that youngsters can use probabilistic information to reduce interference originating from salient but task-irrelevant signals, even though active inhibition is not fully developed. The intensity of inhibition varies as a function of the advance knowledge about an item. Such probabilistic information requires central resources (Watson \& Humphreys, 1997) and influences inhibition. Seemingly, this occurs at an early stage of development and remains stable until adulthood, even though inhibitory control per se is weak in childhood. A second finding, however, suggests that the quantity of visual information available at a given moment is instrumental in the use of probabilistic signals to modulate inhibition. When the search display contained only 6 items, probabilistic knowledge was used efficiently whatever the age and, interestingly, to the same degree. However, when the search display contained 10 items, only participants aged 9 and over made efficient use of probabilistic knowledge. One possible interpretation is that the more items there are to explore, the more central resources are used. And the more that resources are 
allocated to the current stimulus display, the less they are available for the correct use of probabilistic information. Such an interpretation assumes that the quantity of resources required for visual search, inhibition of salient distractors, and the correct use of probabilistic information exceeds the total resources available to 7 and 8 year-olds, and suggests, of course, that the pool of central resources develops during childhood (IrwinChase \& Burns, 2000). Some studies have shown that 10 year-old children and adults use central resources in a similar fashion (Karatekin, 2004; Karatekin et al., 2007). This is in keeping with our own findings, although we believe that whether 9 to 11 year-olds use resources as adults do probably depends on the task at hand (Irwin-Chase \& Burns, 2000).

The overall pattern suggests that inhibition per se develops late, but that the use of probabilistic knowledge develops early in childhood. This use depends, however, on the complexity of the visual stimulus to be processed, suggesting differential use of resources. This adds support to the hypothesis that while inhibition and probabilistic signals are different, they may both depend on central resources.

\section{Theoretical Issues}

As announced in the introductory part, the present series of experiments was constructed on the basis of the Master Activation Map model of attention (MAMm; Michael et al., 2006; Figure 1). This model describes how interactive networks lead to the rise of attentive behavior in adulthood, ie. at the age point of highest efficiency. We would like, therefore, to end with the possibility of using this model and others (e.g., Kahneman, 1973; Posner, 1980; Posner \& Petersen, 1990; Koch \& Ullman, 1985; Theeuwes, 1991; Watson \& Humpreys, 1997) as frameworks for interpreting the trajectories of attentional development observed in this study. 
There would be three subsystems (Michael et al., 2007) that cooperate to produce attention-related phenomena. A computational subsystem made up of maps that process either bottom-up (i.e., generation of salience) or top-down (i.e., activation of relevance) signals. An operatory subsystem made up of the independent and indirectly interacting processes of orienting (comprising engagement, disengagement and movement of attention through space; Posner, 1980) and inhibition. And a directing subsystem that generates and maintains task-related goals and controls the top-down flow of information through capacity limited resources (Kahneman, 1973). In turn, resources regulate inhibition (Watson and Humphreys, 1997). There are a number of interesting questions in this context: How do the postulated processes and their interactions develop through childhood? At what age do they reach the fine-tuned efficiency observed in adults? Do processes such as active inhibition and orienting develop simultaneously? Do their interactions (e.g., maintenance of inhibition during visual search) follow a different developmental pattern? The developmental trends for some components of the MAM model as reflected through our data are summarized in Table 3.

\section{TABLE 3 ABOUT HERE}

Before a location in space containing a salient item can be selected, the differences in feature values of adjacent items must first be computed across all visual dimensions (i.e., colors, sizes, contrasts, etc.) within the primary visual cortex (Li, 2002). The aim is to determine their relative saliencies and arrange them hierarchically and, in parallel, to code the location of these items in the visual field (Koch \& Ullman, 1985). Once the most salient item has been located in space, attention moves towards that location and engages there (Posner, 1980). Orienting of attention is thus driven by the characteristics of external 
events that confer salience. In keeping with the literature (Enns \& Brodeur, 1989; Trick \& Enns, 1998; Donnelly et al., 2007), our results showed that moving attention to the location of the most salient item and engaging it there develops quite early and reaches its high point of efficiency before the age of 7 . This suggests that the underlying computations of salience and localization of the most salient item are also efficient early. At present, there is no consensus on the brain structures that generate salience, but some of the candidate structures, such as the thalamic pulvinar (Robinson \& Petersen, 1992), the primary visual cortex (Li, 2002) and the parietal cortex (Gottlieb, Kusunoki \& Goldberg, 1998) mature early (Casey, Tottenham, Liston et al., 2005). If the salient item is not the target, attention disengages from that location (Posner, 1980) and moves elsewhere, proceeding from item to item until the target is found (Michael \& Gálvez-García, 2011). By contrast, subserved by the parietal cortices that develop slowly, attentional disengagement develops slowly throughout childhood (Waszak, Li \& Hommel, 2010). Our study suggests it starts developing at age 9 and reaches its high point of efficiency at 11 .

Whenever participants select items on the basis of advance knowledge that motivates developing and using incentives, orienting of attention is guided by goals and the internal representations of the target characteristics that confer relevance (Michael et al., 2006) as regards to the requirements of the task at hand. Comparing the baselines of the three Experiments revealed the preparatory effects fully attributable to task instructions (Laberge et al., 2000) and, therefore, to the development of relevance. It is likely that relevance develops before the age of 7 insofar as we found instructions caused participants to search for, and respond to the target more quickly. Interestingly, this effect was found in all age groups and remained virtually unchanged from between the ages of 7 and 11 . It was found to be diminished in adults, suggesting that the point at which it reached its adult level was at some point during adolescence. The recent development of the notion of 
relevance in the literature on attention (Michael et al., 2006; Fecteau \& Munoz, 2006) does not allow for the precise description of the possible underlying neural mechanisms. Michael and colleagues (2006) suggested that relevance would be activated only if participants intend to find the target by using the available knowledge, and later accounts proposed that this could be done through fuzzy logic (Andrieu et al., 2008). The use of such information in order to generate and maintain goals could depend upon subregions of the dorsolateral prefrontal cortex (Michael et al., 2007). The activity of some of these areas seemingly decreases with age (Gogtay et al., 2004), and this may explain the decrement of the effect of probability observed in the performance of the group of adults in this study (see Figure 6B).

In cases where the most salient item of the visual field happens to be the most relevant, as identified through instructions and advance knowledge, a coordination or integration (Fecteau \& Munoz, 2006; Michael et al., 2006; Olk et al., 2008; Ristic \& Kingstone, 2009) of bottom-up (salience) and top-down (relevance) signals takes place. Relevance therefore enhances salient signals (Fecteau \& Munoz, 2006), allowing for faster selection and orienting (Michael et al., 2006) towards the location of the target. Our results suggest children as young as 7 already use advance knowledge to orient attention towards salient items when the probability of being the target is very high. Yet, their use of such incentives seems to reach adult levels at age 8 , suggesting that the age at which they start to develop may be before 7. This is in keeping with the study by Ristic and Kingstone (2009) who found that preschool children (3-6 years old) exhibited an immature integration of what we call salience and relevance.

At this point, we may tentatively conclude that the components of the computational subsystem as conceived within the MAMm (Michael et al., 2007) develop through 
childhood at different rates, and this may be linked to differences in maturation of the cerebral tissue that subserves such computations (Casey et al., 2005). The computation of salience (Koch \& Ullman, 1985) develops well before the full development of relevance, and this matches previous findings as well as the assumption that salience and relevance are independent. In contrast to the assumption that their integration is efficient when orienting based on relevance is fully developed (Ristic \& Kingstone, 2009), we found that such an integration follows a different developmental trajectory (asymptote reached at age 8) to relevance (asymptote reached after the age of 11). Such a finding supports the idea that integrated activities depend of course on their components (i.e., salience and relevance) and may be highly correlated to them, although the fact that their developmental sequences are separate suggests they acquire an independent status (Fecteau \& Munoz, 2006; Michael et al., 2006; 2007).

Searching for a target in presence of a salient distractor requires continuous inhibition of the signals that define it as salient, and since the inflow of visual information is continuous, inhibition must be maintained until the target is found. However, the results of our study suggest that when much is known in advance about the task-irrelevant salient item, its presence slows chronometric performance and its effects remain virtually unchanged up to the age of 11. A reliable decrement of this cost is observed in adulthood, suggesting that inhibitory processes that act to reduce it are mastered at some point during adolescence. This is consistent with most studies on the developmental trajectory of inhibition and the maturation of some areas of the right ventrolateral prefrontal cortex from which inhibition is thought to depend (e.g., Levin et al., 1991; Bunge et al., 2002; Brocki \& Bohlin, 2004; Fan, McCandliss, Fossella et al., 2005, Aron et al., 2007; Michael et al., 2001b; 2006; Waszak et al., 2010). 
Here, a conclusion can be drawn about the operatory subsystem as conceived within the MAMm (Michael et al., 2006; Michael et al., 2007). The results show that the operations involved in the orienting process (move, engage, disengage; Posner, 1980) follow different developmental trajectories to the inhibition process. This is in agreement with behavioral (Waszak et al., 2010) neuroanatomical findings (e.g., Fan et al., 2005). Some differences in maturational rate between the parietal cortices subserving orienting and the ventrolateral frontal cortex subserving inhibitory activity have already been described (Casey et al., 2005; Bunge et al., 2002; Huttenlocher, 1979).

The control units of top-down activities, defined as part of the directing subsystem (Michael et al., 2007), were not targeted by this study. There is, however, some indirect evidence about their functioning. For instance, the effects of salient distractors on chronometric performance were reduced across all age groups and to a similar degree when participants were informed of the high probability that the salient item was a distractor, suggesting that the use of probabilities to activate inhibition is already mature before the age of 7 . Furthermore, probabilistic information seems more resourceconsuming up to the age of 8 , so that the processing of additional items exceeds the resources available, causing the unsuccessful use of such information (Kahneman, 1973). However, these are isolated findings, and further investigation is needed before the developmental trajectories of the directing subsystem be defined. 


\section{References}

Akhtar, N., \& Enns, J. T. (1989). Relations between covert orienting and filtering in the development of visual attention. Journal of Experimental Child Psychology, 48, 315334.

Andrieu, Q., Dehais, F., Izaute, A., Lesire, C., Tessier, C. (2008). Towards a dynamic computational model of visual attention International Conference on Humans Operating Unmanned Systems (HUMOUS'08), Brest, France.

Aron, A.R. (2007). The neural basis of inhibition in cognitive control. Neuroscientist, 13, 214-228.

Aron, A.R. (2004) Aron, A. R., Robbins, T. W., Poldrack, R. A. (2004). Inhibition and the right inferior frontal cortex. Trends in Cognitive Sciences, 8, 170-177.

Aron, A. R., Fletcher, P. C., Bullmore, E. T., Sahakian, B. J., Robbins, T. W. (2003). Stopsignal inhibition disrupted by damage to right inferior frontal gyrus in humans. Nature Neuroscience, 6, 115-116.

Bedard, A. C., Nichols, S., Barbosa, J. A., Schachar, R., Logan, G. D., and Tannock, R. (2002). The development of selective inhibitory control across the life span. Developmental Neuropsychology, 21, 93-111.

Bjorklund, D. F., Harnishfeger, K. (1990). The resources construct in cognitive development : diverses sources of evidence and theory of inefficient inhibition. Developmental Review, 10, 48-71.

Brocki, K. C., \& Bohlin, G. (2004). Executive Functions in children aged 6-13: A dimensional and developmental study. Developmental Neuropsychology, 26, 571-593.

Brodeur, D., \& Boden, C. (2000). The effects of spatial uncertainty and cue predictability on visual orienting in children. Cognitive Development, 15, 367-382. 
Bunge, S. A., Dudukovic, N. M., Thomason, M. E., Vaidya, C. J. \& Gabrieli, J. D. (2002). Immature frontal lobe contributions to cognitive control in children: evidence from fMRI. Neuron, 33, 301-311.

Carver, A. C., Livesey, D. J., \& Charles, M. (2001). Age related changes in inhibitory control as measured by stop signal task performance. International Journal of Neuroscience, 107(1-2), 43-61.

Casey, B.J., Tottenham, N., Liston C. \& Durston, S. (2005). Imaging the developing brain: What we have learned about cognitive development? Trends in Cognitive Sciences, 9, 104-110.

Comalli, P. E., Wapner, S., and Werner, H. (1962). Interference effects of Stroop colorword test in childhood, adulthood and aging. Journal of Genetic Psychology, 100, 4753.

Davidson, M., Amso, D., Cruess Anderson, L., Diamond, A. (2006). Development of cognitive control and executive functions from 4 to 13 years: Evence from manipulations of memory, inhibition, and task switching. Neuropsychologia, 44, 20372078.

Dempster, F. N. (1993). Resistance to interference: Developmental changes in a basic processing mechanism. In M. L. Howe and R. Pasnak (Eds.), Emerging themes in cognitive development, Vol. 1: Foundations (pp. 3-27). New York: Springer.

Desimone, R., Duncan, J. (1995). Neural mechanisms of selective attention. Annual Review of Neuroscience, 18, 193-222.

Donnelly, N., Cave, K., Greenway, R., Hadwin, J. A., Stevenson, J., \& Sonuga-Barke, E. (2007). Visual search in children and adults: top-down and bottom-up mechanisms. The Quarterly Journal of Experimental Psychology, 60(1), 120-36. 
Duncan, J., \& Humphreys, G. W. (1989). Visual search and stimulus similarity. Psychological Review, 96, 433-458.

Enns, J. T., \& Akhtar, N. (1989). A developmental study of filtering in visual attention. Child Development, 60, 1188-1199.

Enns, J. T. (1993). What can be learnt about attention from studying its development? Canadian Psychology, 34, 271-281.

Enns, J., \& Brodeur, D. (1989). A developmental study of covert orienting to peripheral visual cues. Journal of Experimental Child Psychology, 48, 171-189.

Enns, J. T. \& Cameron, S. (1987). Selective attention in young children: The relations between visual search, filtering, and priming. Journal of Experimental Child Psychology, 44, 38-63.

Enns, J. T., \& Girgus, J. S. (1985). Developmental changes in selective and integrative visual attention. Journal of Experimental Child Psychology, 40, 319-337.

Eriksen, B. A., \& Eriksen, C. W. (1974). Effects of noise letters on the identification of a target letter in a nonsearch task. Perception \& Psychophysics, 16, 143-149.

Eriksen, C. W., \& St. James, J. D. (1986). Visual attention within and around the field of focal attention: A zoom lens model. Perception and Psychophysics, 40, 225-240.

Fan, J., McCandliss, B., Fossella, J., Flombaum, J., Posner, M. (2005). The activation of attentional networks. Neurolmage, 26, 471-479.

Facoetti, A., \& Molteni, M. (2000). Is attentional focusing an inhibitory process at distractor location? Cognitive Brain Research, 10, 185-188.

Fecteau, J. H. and Munoz, D. P. (2006). Salience, relevance, and spiking neurons: a priority map governs target selection. Trends in Cognitive Sciences, 10, 382-390. 
Goldberg, M., Maurer, D., \& Lewis, T. L. (2001). Developmental changes in attention: The effects of endogenous cueing and of distractors. Developmental Science, 4, 209-219.

Goldman, K. J., Flanagan, T., Shulman, C., Enns, J. T., \& Burack, J. A. (2005). Voluntary orienting among children and adolescents with Down syndrome and MA-matched typically developing children. American Journal on Mental Retardation, 110, 157-163.

Gogtay, N., Giedd, J., Lusk, L., et al. (2004) Dynamic mapping of human cortical development during childhood through early adulthood. Proc. Natl. Acad. Sci. U. S. A., $101,8174-8179$

Gottlieb, J.P., Kusunoki, M., Goldberg, M.E. (1998). The representation of visual salience in monkey parietal cortex. Nature, 391, 481-484.

Gupta, R. \& Kar, B. R. (2009). Development of attentional processes in children with ADHD and normally developing children. Progress in Brain Research, 176, 259-276.

Hommel, B., Li, Z. H., \& Li, S. -C. (2004). Visual search across the life span. Developmental Psychology, 40, 545-558.

Huttenlocher, P. (1979). Synaptic density in human frontal cortex: Developmental changes and effects of aging. Brain Research, 163, 195-205.

larocci, G., Enns, J. T., Randolph, B., \& Burack, J. A. (2009). The modulation of visual orienting reflexes across the lifespan. Developmental Science, 12(5), 715-724.

Irwin-Chase, H. \& Burns, B. (2000). Developmental changes in children's abilities to share and allocate attention in a dual task. Journal of Experimental Child Psychology, 77(1), 61-85.

Johnstone, S. J., Dimoska, A., Smith, J. L., Barry, R. J., Pleffer, C. B., Chiswick, D. \& Clarke, A. R. (2007). The development of stop-signal and Go/Nogo response inhibition 
in children aged 7-12 years: performance and event-related potential indices. International Journal of Psychophysiology, 63, 25-38.

Kaye, B., \& Ruskin, E. (1990). The development of attentional control mechanisms. In J. T. Enns (Ed.), The development of attention: Research and theory (pp. 227-244). Amsterdam: Elsevier.

Klein, R. M. (1988). Inhibitory tagging system facilitates visual search. Nature, 334, 430431.

Klein, R. M. (2000). Inhibition of return. Trends in Cognitive Sciences, 4, 138-147.

Koch, C. \& Ullman, S. (1985). Shifts in selective visual attention : towards the underlying neural circuitry. Human Neurobiology, 4, 219-227.

Kok, A. (1999). Varieties of inhibition inhibition: manifestations in cognition, event-related potentials and aging. Acta Psychologica, 101, 129-158.

LaBerge, D. (1983). Spatial extent of attention to letters in words. Journal of Experimental Psychology: Human Perception \& Performance, 9, 371-379.

LaBerge, D. (1995). Attentional processing: The brain's art of mindfulness. Cambridge, MA: Harvard University Press.

LaBerge, D., Auclair, L., \& Siéroff, E. (2000). Preparatory attention : Experiment and theory. Consciousness \& Cognition, 9, 396-434.

Levin, H. S., Culhane, K. A., Jartmann, J., Evankovich, K., Mattson, A. J., Harward, J., Gingholz, G., Ewing-Cobbs, L., \& Fletcher, J. M. (1991). Developmental changes in performance on tests of purported frontal lobe functioning. Developmental Neuropsychology, 7, 377-395.

Li, Z. (2002). A saliency map in primary visual cortex. Trends in Cognitive Sciences, 6, 916. 
Michael, G. A., Kleitz, C., Sellal F., Hirsch, E., \& Marescaux, C. (2001b). Controlling attentional priority by preventing changes in oculomotor programs: a job for the premotor cortex? Neuropsychologia, 39, 1112-1120.

Michael, G. A., Gálvez-García, G. (2011). Salience-based progression of visual attention. Behavioural Brain Research, 224, 87-99.

Michael, G. A., Garcia, S., Fernandez, D., Sellal, F., \& Boucart, M. (2006). The ventral premotor cortex (vPM) and resistance to interference. Behavioral Neuroscience, 20, 442-467.

Michael, G. A., Buron V. (2005). The human pulvinar and stimulus-driven attentional control. Behavioral Neuroscience, 119, 1353-1367.

Michael, G.A., Vairet, A. -V., \& Fernandez, D. (2007). Capture attentionnelle en vision: La saillance, la pertinence, et la balance cortico-sous-corticale, In G.A. Michael (Ed.) Neuroscience cognitive de l'attention visuelle (pp. 165-201). Marseille: Solal.

Norman, D.A., \& Borbow, D.G. (1975). On data-limited ans resource-limited processes. Cognitive Psychology, 7, 44-64.

Olk, B., Cameron, B., \& Kingstone, A. (2008). Enhanced orienting effects: evidence for an interaction principle. Visual Cognition, 16, 979-1000.

Oosterlaan, J., \& Sergeant, J. A. (1996). Inhibition in ADHD, aggressive, and anxious children: A biologically based model of child psychopathology. Journal of Abnormal Child Psychology, 24 (1), 19-36.

Passler, P. A., Isaac, W., Hynd, G. W. (1985). Neuropsychological development of behavior attributed to frontal lobe functioning in children, Developmental Neuropsychology, 4, 349-370. 
Peelen, M. V., Heslenfeld, D. J., \& Theeuwes, J. (2004). Endogenous and exogenous attention shifts are mediated by the same large-scale neural network. Neurolmage, 22, 822-830.

Posner, M. I., Cohen, Y. (1984). Components of visual orienting. In H. Bouma \& D. G. Bouwhuis (Eds.), Attention and performance $X$ (pp. 531-556), Hillsdale, N.J.: Erlbaum.

Posner, M. (1980). Orienting of attention. Quarterly journal of experimental psychology, 32, $3-25$.

Ridderinkhof, K. R. \& Van der Molen, M. W. (1995). A psychophysiological analysis of developmental differences in the ability to resist interference. Child Development, 66, 1040-1056.

Ristic, J., \& Kingstone, A. (2009). Rethinking attentional development: Reflexive and volitional orienting in children and adults. Developmental Science, 12, 289-296.

Ristic, J., Friesen, C. K., \& Kingstone, A. (2002). Are eyes special? It depends on how you look at it. Psychonomic Bulletin \& Review, 9, 507-513.

Robinson, DL, Petersen, S. (1992). The pulvinar and visual salience. Trends in Neuroscience, 15, 127-132.

Schachar, R., \& Logan, G. D. (1990). Impulsivity and inhibitory control in normal development and childhood psychopathology. Developmental Psychology, 26, 710720.

Theeuwes, J. (1991). Exogenous and endogenous control of attention: The effect of visual onsets and offsets. Perception \& Psychophysics, 49, 83-90.

Theeuwes, J. (1992). Perceptual selectivity for color and form. Perception \& Psychophysics, 51, 599-606. 
Theeuwes, J. (1995). Temporal and spatial characteristics of preattentive and attentive processing. Visual Cognition, 2, 221-233.

Tipples, J. (2002). Eye gaze is not unique: Automatic orienting in response to uninformative arrows. Psychonomic Bulletin \& Review, 9, 314-318.

Treisman, A., \& Gelade, G. (1980). A feature-integration theory of attention. Cognitive Psychology, 12, 97-136.

Trick, L. M., \& Enns, J. T. (1998). Life-span changes in attention: The visual search task. Cognitive Development, 13(3), 369-386

Van der Lubbe, R. H. J., \& Keuss, P. J. G. (2001). Focused attention reduces the effect of lateral interference in multi-element arrays. Psychological Research, 65, 107-118.

Wager, T. D., Sylvester, C. -Y. C., Lacey, S., Nee, D. E., Franklin M., \& Jonides, J. (1995). Common and unique components of response inhibition revealed by fMRI. Neurolmage, 27, 323-340.

Wainwright, A., Bryson, S. (2002). The development of exogenous orienting: mechanisms of control. Journal of Experimental Child Psychology, 82(2), 141-155.

Wainwright, A., \& Bryson, S. E. (2005). The development of endogenous orienting: Control over the scope of attention and lateral asymmetries. Developmental Neuropsychology, 27, 237-255.

Waszak, F., Li, S.-C., \& Hommel, B. (2010). The development of attentional networks: Cross-sectional findings from a life span sample. Developmental Psychology, 46, 337349.

Watson, D. G., \& Humphreys, G. W. (1997). Visual marking: Prioritizing selection for new objects by top-down attention inhibition of old objects. Psychological Review, 104, 90122. 
Williams, B. R., Ponesse, J. S., Schachar, R. J., Logan, G. D., \& Tannock, R. (1999). Development of inhibitory control across the life span. Developmental Psychology, 35, $205-213$.

Wise, L. A., Sutton, J. A. \& Gibbons, P. D. (1975). Decrement in Stroop interference time with age. Perceptual and Motor Skills, 41, 149-150.

Wolfe, J. M., Cave, K. R., \& Franzel, S. L. (1989). Guided search: An alternative to the feature integration model for visual search. Journal of Experimental Psychology: Human Perception \& Performance, 15, 419-433.

Xue, G., Aron, A. R., Poldrack, R. A. (2008). Common neural substrates for inhibition of spoken and manual responses. Cerebral Cortex, 18, 1923-1932.

Xue, G., Lu, Z., Levin, I. P., Weller, J. A., Li, X., \& Bechara, A. (2009). Functional dissociations of risk and reward processing in the medial prefrontal cortex. Cerebral Cortex, 19, 1019-1027.

Yantis, S. (1998). Control of visual attention. In H. Pashler (Ed.) Attention (pp.223-256). Hove: Psychology Press.

Yantis, S., \& Johnston, J. C. (1990). On the locus of visual selection: Evidence from focused attention tasks. Journal of Experimental Psychology: Human Perception and Performance, 16, 135-149.

Yantis, S., Jonides J. (1984). Abrupt visual onsets and visual selective attention: evidence from visual search. Journal of Experimental Psychology: Human Perception and Performance, 10, 601-621. 


\section{Footnotes}

1. The difference between the analogue conditions in the classical Posner's paradigm (i.e., invalid and valid) are taken as reflecting orienting of attention. Within the frames of a modified visual search paradigm, this difference cannot be interpreted as such.

\section{Authors' Note}

Part of this work was supported by two grants from the French ANR (Agence Nationale de la Recherche) to George Michael (ANR BLAN07-3-203520) and Bernard Lété (ANR-06APPR-005), and by one regional grant to Stéphanie Ducrot (Région PACA) and one to George Michael (Cluster 11 - Région Rhône-Alpes).

The authors are grateful to Solveig Relland for her technical assistance. 
Table 1. Mean RT (1 SD) observed across the six age groups in the three Experiments as a function of the tested condition.

Age Group

\begin{tabular}{|c|c|c|c|c|c|c|}
\hline & $\begin{array}{c}7 \\
N=74\end{array}$ & $\begin{array}{c}8 \\
N=100\end{array}$ & $\begin{array}{c}9 \\
N=93\end{array}$ & $\begin{array}{c}10 \\
N=80\end{array}$ & $\begin{array}{c}11 \\
N=77\end{array}$ & $\begin{array}{c}20 \\
N=74\end{array}$ \\
\hline \multicolumn{7}{|l|}{ Experiment 1} \\
\hline 6 items & $1518(480)$ & $1240(287)$ & $1089(217)$ & $993(229)$ & $915(160)$ & $782(109)$ \\
\hline 10 items & $1579(602)$ & $1279(341)$ & $1091(236)$ & $1002(267)$ & $926(177)$ & $771(114)$ \\
\hline Mean & $1549(520)$ & $1260(292)$ & $1090(201)$ & $998(241)$ & $921(158)$ & $777(107)$ \\
\hline \multicolumn{7}{|l|}{ Salient Distractor } \\
\hline 6 items & $2349(607)$ & $2083(582)$ & $1809(460)$ & $1534(319)$ & $1517(362)$ & $1142(186)$ \\
\hline 10 items & $2815(809)$ & $2332(621)$ & $2068(584)$ & $1904(474)$ & $1800(507)$ & $1332(236)$ \\
\hline Mean & $2582(638)$ & $2208(542)$ & $1939(491)$ & $1720(370)$ & 1659 (413) & 1237 (197) \\
\hline \multicolumn{7}{|l|}{ Baseline } \\
\hline 6 items & $2073(662)$ & $1643(496)$ & $1387(351)$ & $1239(275)$ & $1194(350)$ & $894(165)$ \\
\hline 10 items & $2480(723)$ & 2131 (713) & $1796(564)$ & $1569(456)$ & $1475(379)$ & $1120(293)$ \\
\hline Mean & $2277(624)$ & $1887(549)$ & $1592(413)$ & $1405(332)$ & $1335(322)$ & 1008 (209) \\
\hline \multicolumn{7}{|l|}{$\begin{array}{r}\text { Experiment } \mathbf{2} \\
\text { Salient Target }\end{array}$} \\
\hline 6 items & $1326(329)$ & $1098(272)$ & $954(180)$ & $865(170)$ & $833(203)$ & $647(85)$ \\
\hline 10 items & 1367 (355) & 1130 (309) & $979(184)$ & $913(238)$ & $852(217)$ & $662(91)$ \\
\hline Mean & 1347 (319) & 1114 (277) & $967(164)$ & $889(192)$ & $843(204)$ & $655(86)$ \\
\hline \multicolumn{7}{|l|}{ Baseline } \\
\hline 6 items & $2013(601)$ & $1609(535)$ & $1349(408)$ & $1196(272)$ & $1163(360)$ & $853(120)$ \\
\hline 10 items & $2254(732)$ & $1863(614)$ & $1570(536)$ & $1398(351)$ & $1288(324)$ & $1050(233)$ \\
\hline Mean & $2134(625)$ & $1736(542)$ & $1460(418)$ & $1297(280)$ & 1226 (315) & 952 (159) \\
\hline \multicolumn{7}{|l|}{ Experiment 3} \\
\hline 6 items & 2237 (569) & $1980(630)$ & $1613(392)$ & $1485(418)$ & $1379(392)$ & $994(168)$ \\
\hline 10 items & $2809(840)$ & $2360(761)$ & $1982(551)$ & $1749(534)$ & 1621 (424) & 1201 (275) \\
\hline Mean & $2524(646)$ & $2171(652)$ & $1798(446)$ & $1617(456)$ & 1501 (383) & $1098(200)$ \\
\hline \multicolumn{7}{|l|}{ Baseline } \\
\hline 6 items & $2089(589)$ & $1698(607)$ & $1424(351)$ & $1251(386)$ & $1161(311)$ & $893(158)$ \\
\hline 10 items & $2564(825)$ & 2080 (779) & $1692(573)$ & $1503(444)$ & 1431 (466) & 1047 (219) \\
\hline Mean & $2327(640)$ & $1890(654)$ & $1558(435)$ & $1378(396)$ & 1297 (359) & $970(173)$ \\
\hline
\end{tabular}


Table 2. Mean number of errors (1 SD) observed across the six age groups in the three Experiments as a function of the tested condition.

Age Group

\begin{tabular}{|c|c|c|c|c|c|c|}
\hline & $\begin{array}{c}7 \\
N=74\end{array}$ & $\begin{array}{c}8 \\
N=100\end{array}$ & $\begin{array}{c}9 \\
N=93\end{array}$ & $\begin{array}{c}10 \\
N=80\end{array}$ & $\begin{array}{c}11 \\
N=77\end{array}$ & $\begin{array}{c}20 \\
N=74\end{array}$ \\
\hline \multicolumn{7}{|l|}{ Experiment 1} \\
\hline 6 items & $1,18(2,0)$ & $0,72(1,4)$ & $1,06(1,6)$ & $0,7(1,1)$ & $0,5(1,1)$ & $0,0(0,2)$ \\
\hline 10 items & $1,0(1,6)$ & $0,69(1,3)$ & $1,01(1,9)$ & $0,73(1,5)$ & $0,6(1,3)$ & $0,0(0,2)$ \\
\hline Mean & $1,09(1,7)$ & $0,70(1,2)$ & $1,03(1,6)$ & $0,7(1,2)$ & $0,6(1,1)$ & $0,0(0,2)$ \\
\hline \multicolumn{7}{|l|}{ Salient Distractor } \\
\hline 6 items & $1,58(2,0)$ & $1,2(2,0)$ & $1,48(1,9)$ & $0,88(1,4)$ & $0,88(1,2)$ & $0,13(0,3)$ \\
\hline 10 items & $1,81(2,6)$ & $1,24(1,8)$ & $1,65(2,1)$ & $1,11(1,6)$ & $0,89(1,3)$ & $0,21(0,4)$ \\
\hline Mean & $1,69(2,2)$ & $1,22(1,8)$ & $1,56(1,8)$ & $1,0(1,4)$ & $0,8(1,1)$ & $0,1(0,2)$ \\
\hline \multicolumn{7}{|l|}{ Baseline } \\
\hline 6 items & $1,20(1,7)$ & $0,84(1,3)$ & $1,19(2,0)$ & $0,7(1,3)$ & $0,76(1,2)$ & $0,1(0,3)$ \\
\hline 10 items & $1,20(1,6)$ & $0,9(1,2)$ & $1,05(1,5)$ & $0,83(1,6)$ & $0,79(1,3)$ & $0,16(0,4)$ \\
\hline Mean & $1,20(1,4)$ & $0,87(1,1)$ & $1,12(1,6)$ & $0,76(1,3)$ & $0,77(1,1)$ & $0,14(0,2)$ \\
\hline \multicolumn{7}{|l|}{ Experiment 2} \\
\hline \multicolumn{7}{|l|}{ Salient Target } \\
\hline 6 items & $0,85(1,2)$ & $0,77(1,1)$ & $1,0(1,5)$ & $0,7(1,1)$ & $0,6(1,1)$ & $0,2(0)$, \\
\hline 10 items & $0,66(1,1)$ & $0,62(1,2)$ & $0,8(1,4)$ & $0,6(1,0)$ & $0,6(1,0)$ & $0,1(0)$, \\
\hline Mean & $0,75(1,1)$ & $0,69(1,0)$ & $0,92(1,3)$ & $0,71(0,9)$ & $0,66(0,9)$ & $0,18(0,3)$ \\
\hline \multicolumn{7}{|l|}{ Baseline } \\
\hline 6 items & $1,24(1,8)$ & $0,69(1,1)$ & $1,12(1,9)$ & $0,86(1,4)$ & $0,72(1,5)$ & $0,1(0,4)$ \\
\hline 10 items & $1,21(1,8)$ & $1,11(1,7)$ & $1,02(1,6)$ & $0,71(1,4)$ & $0,76(1,3)$ & $0,08(0,3)$ \\
\hline Mean & $1,22(1,7)$ & $0,9(1,3)$ & $1,07(1,6)$ & $0,78(1,2)$ & $0,74(1,2)$ & $0,1(0,2)$ \\
\hline \multicolumn{7}{|l|}{ Experiment 3} \\
\hline \multicolumn{7}{|l|}{ Salient Distractor } \\
\hline 6 items & $1,71(2,4)$ & $1,34(1,9)$ & $1,29(1,9)$ & $1,07(1,4)$ & $0,90(1,5)$ & $0,2(0,4)$ \\
\hline 10 items & $1,47(2,3)$ & $1,06(1,6)$ & $1,36(2,1)$ & $0,91(1,7)$ & $0,94(1,7)$ & $0,18(0,3)$ \\
\hline Mean & $1,59(2,2)$ & $1,2(1,6)$ & $1,32(1,9)$ & $0,99(1,4)$ & $0,92(1,5)$ & $0,19(0,2)$ \\
\hline \multicolumn{7}{|l|}{ Baseline } \\
\hline 6 items & $1,04(1,5)$ & $0,91(1,5)$ & $0,89(1,3)$ & $0,68(1,2)$ & $0,63(1,2)$ & $0,1(0,4)$ \\
\hline 10 items & $1,13(1,9)$ & $0,82(1,3)$ & $1,07(1,9)$ & $0,72(1,3)$ & $0,68(1,2)$ & $0,09(0,2)$ \\
\hline Mean & $1,08(1,6)$ & $0,86(1,3)$ & $0,9(1,5)$ & $0,7(1,2)$ & $0,6(1,1)$ & $0,1(0)$, \\
\hline
\end{tabular}


Table 3. Developmental trends observed in the three experiments for each component of the MAM model.

\begin{tabular}{|c|c|c|}
\hline Component & $\begin{array}{c}\text { Change } \\
\text { Point (age) }\end{array}$ & $\begin{array}{c}\text { Asymptote } \\
\text { (age) }\end{array}$ \\
\hline \multicolumn{3}{|l|}{ Computational subsystem } \\
\hline salience & $<7$ & 7 \\
\hline relevance & $<7$ & $>11$ \\
\hline integration of salience and relevance (MAM) & $<7$ & 8 \\
\hline \multicolumn{3}{|l|}{ Operatory subsystem } \\
\hline - move and engage & $<7$ & 7 \\
\hline - disengage & 9 & 11 \\
\hline Inhibition & $>11$ & $>11$ \\
\hline \multicolumn{3}{|l|}{ Directing subsystem } \\
\hline use of incentives to inhibit & 7 & 7 \\
\hline control of resources to inhibit & 9 & 10 \\
\hline
\end{tabular}




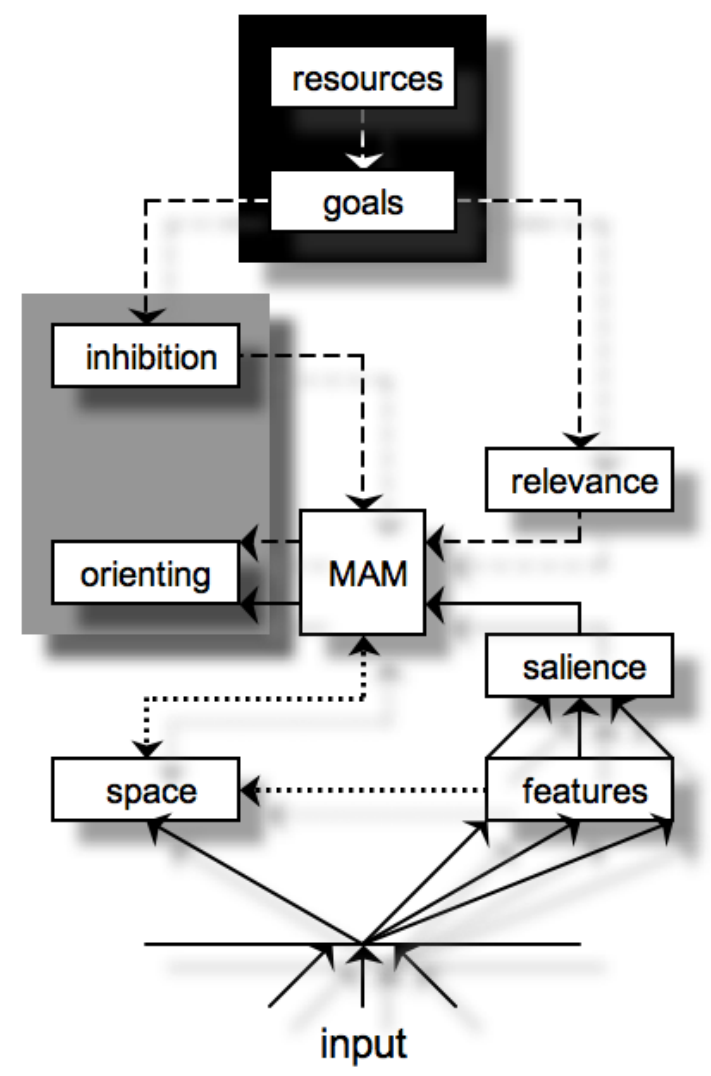

Figure 1. The Master Activation Map model by Michael et al. (2006). 


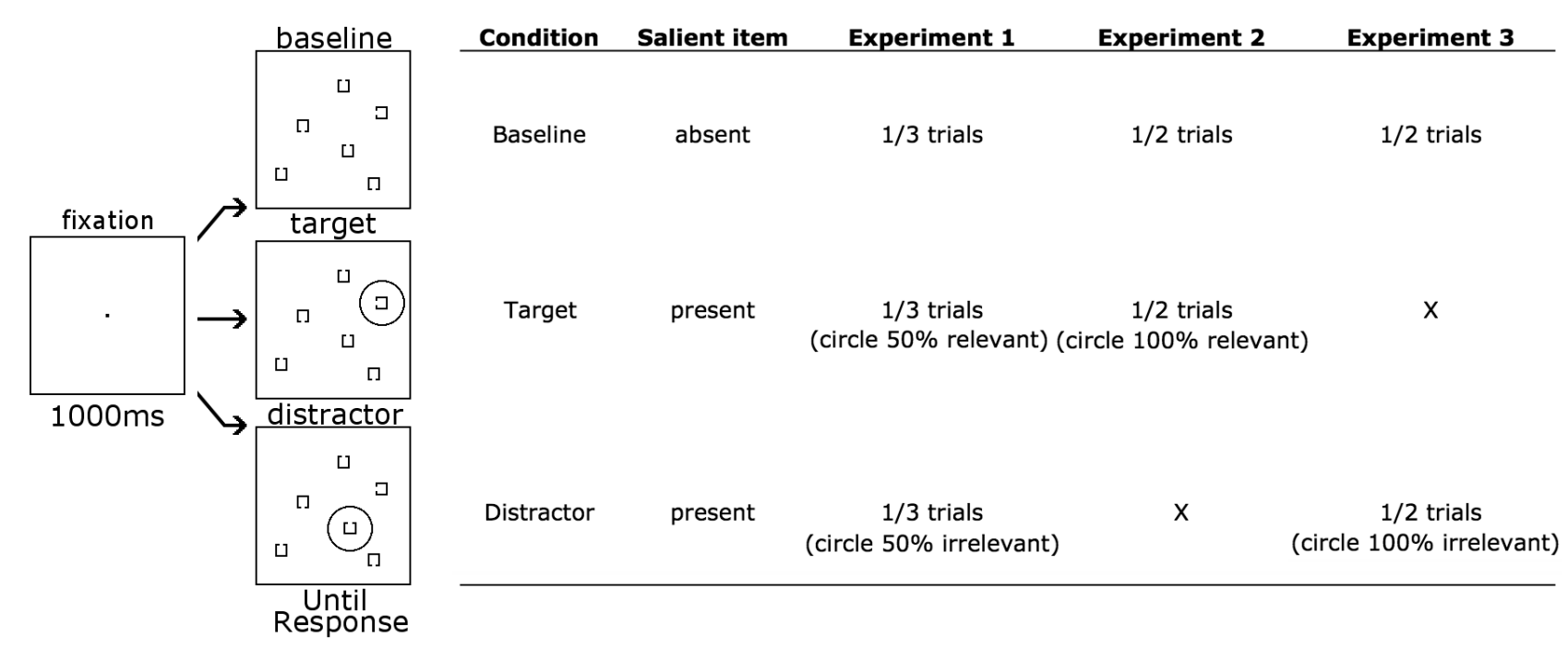

Figure 2. Description of the protocol used in the present study. The probability of the presence of the salient item and of its probability to be associated to the target varied in each Experiment: $1 / 3$ of trials and $50 \%$ relevant in Experiment 1, 1/2 of trials and $100 \%$ relevant in Experiment 2, and 1/2 of trials and 100\% irrelevant in Experiment 3. The terms 'relevant' and 'irrelevant' refer as to whether the circle contained the target or not, respectively. 

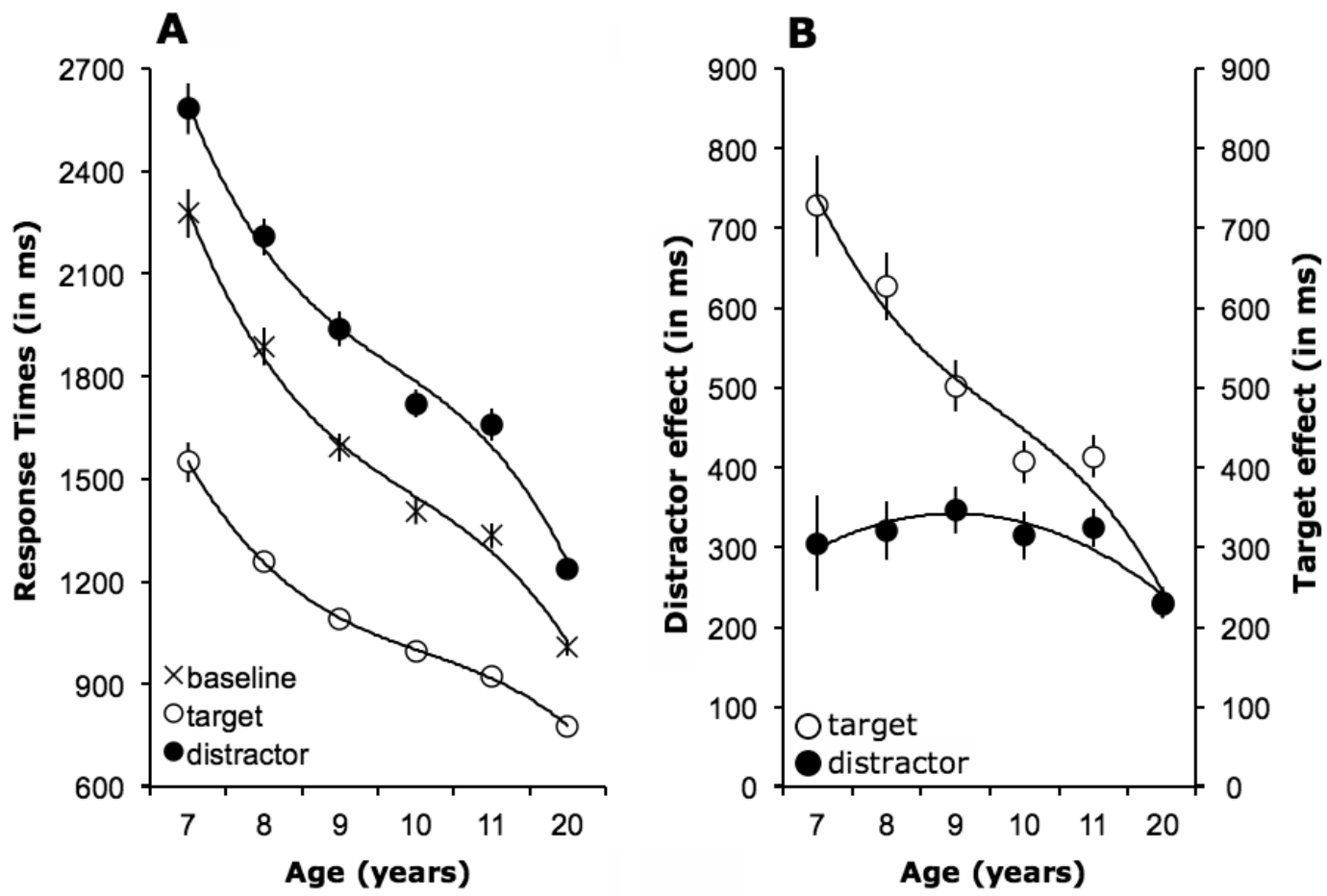

Figure 3. Experiment 1. (A) Mean RT and 1 SEM as a function of age and target condition (baseline: no salient item; target: salient item $50 \%$ task relevant ; distractor: salient item $50 \%$ task irrelevant). (B) Mean and 1 SEM of target effect (baseline condition minus target condition) and distractor effect (distractor condition minus baseline condition). 


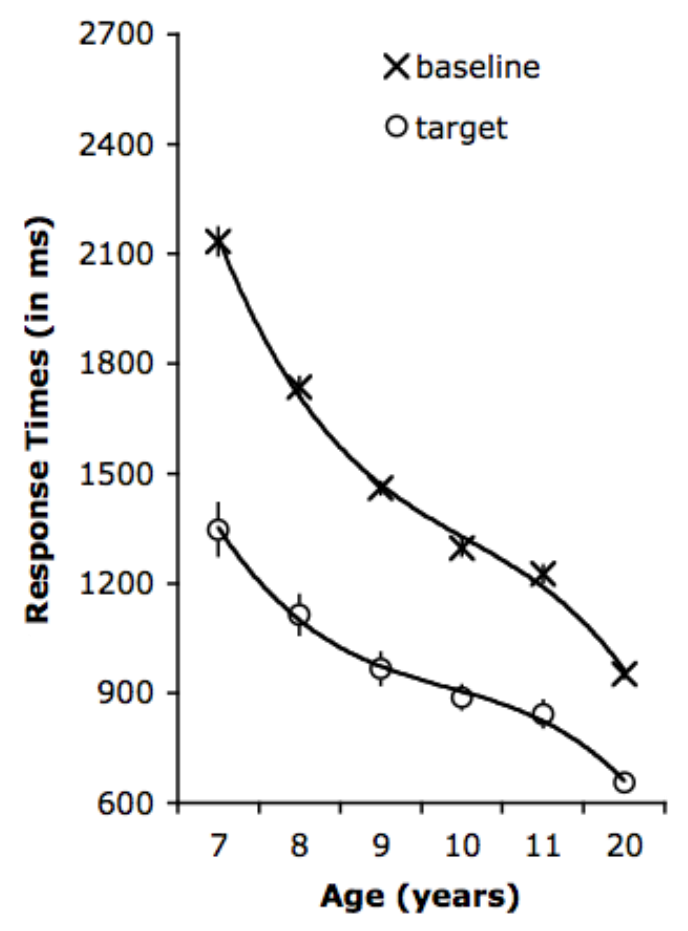

Figure 4. Experiment 2. Mean RT and 1 SEM as a function of age and target condition (baseline: no salient item; target: salient item 100\% task relevant). 

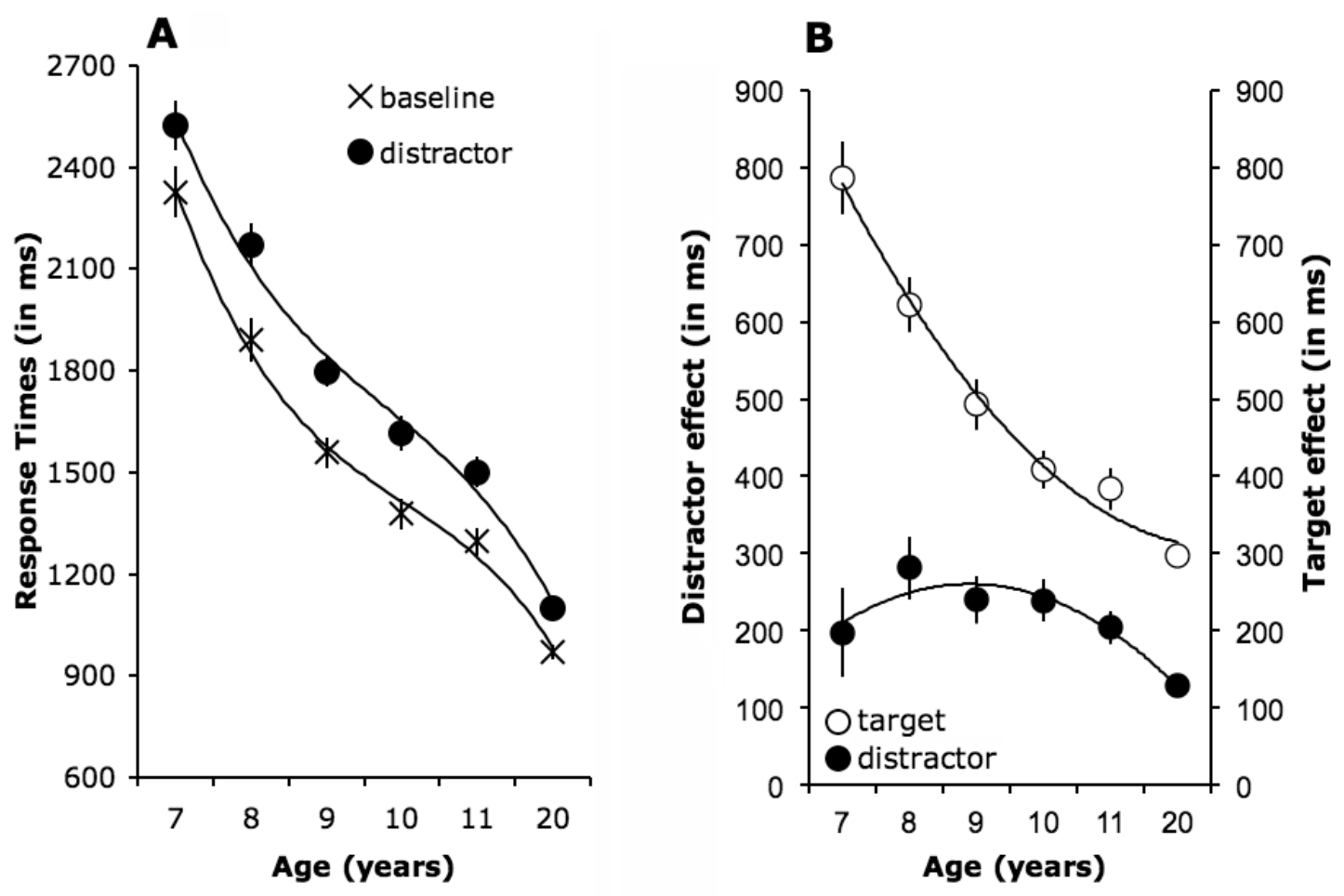

Figure 5. (A) Mean RT and 1 SEM as a function of age and target condition (baseline: no salient item; distractor: salient item 100\% task irrelevant) observed in Experiment 3. (B) Mean and 1 SEM of the target effect (target condition minus baseline condition) and the distractor effect (distractor condition minus baseline condition) observed in Experiments 2 and 3 , respectively. Note that the scale is not the same for the target and the distractor effects for reasons of visibility. 

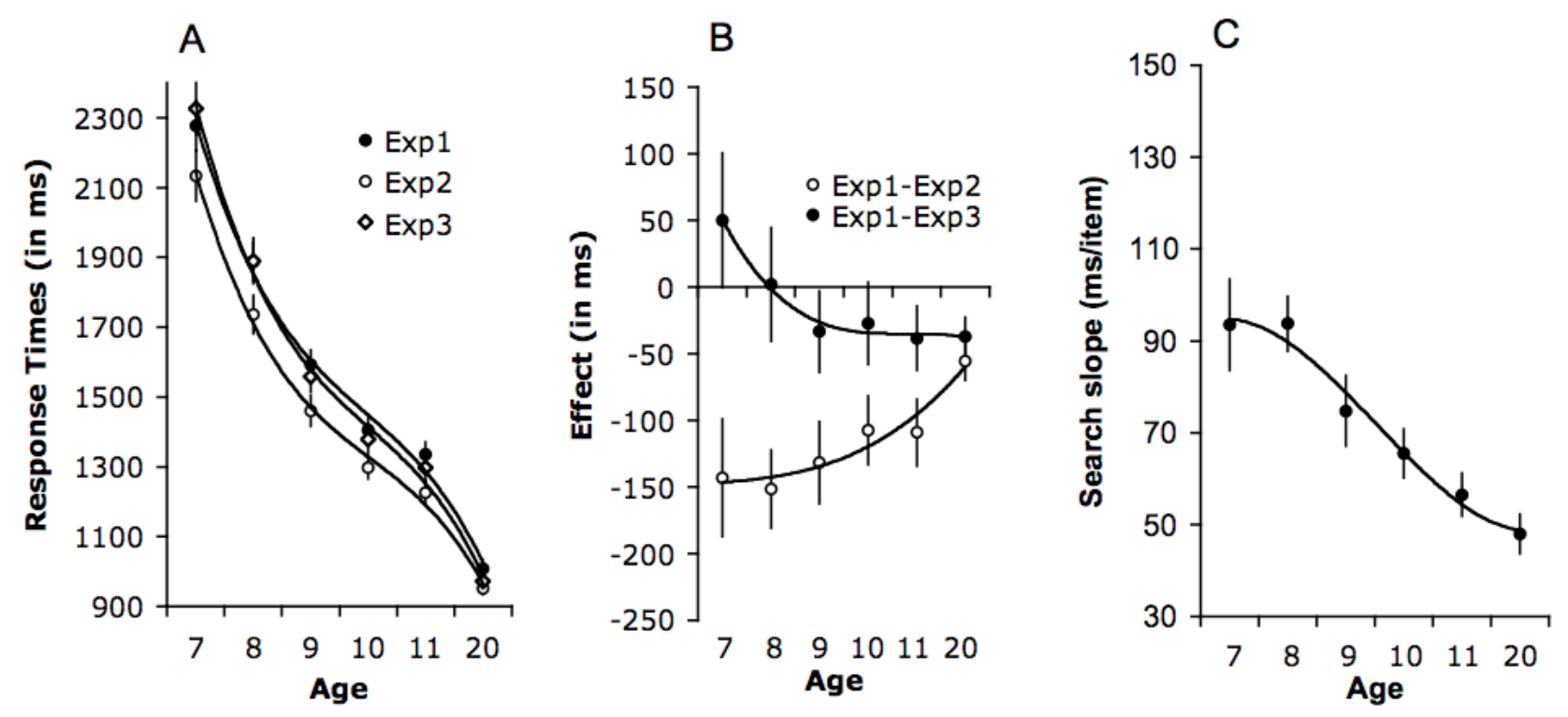

Figure 6. Between-Experiment analysis of baseline conditions. (A) Mean RT and 1 SEM as a function of age and experimental context. (B) Mean and 1 SEM effect of experimental context: effect of knowing that the salient item, whenever present, is always associated with the target (Exp.1 minus Exp. 2), and effect of knowing that the salient item, whenever present, is always associated with a distractor (Exp.1 minus Exp. 3). (C) Mean and 1 SEM search slopes obtained in the three Experiments, expressed in milliseconds per item. Note that the knowledge about the salient target influenced RT in the baselines where no salient item was presented. 

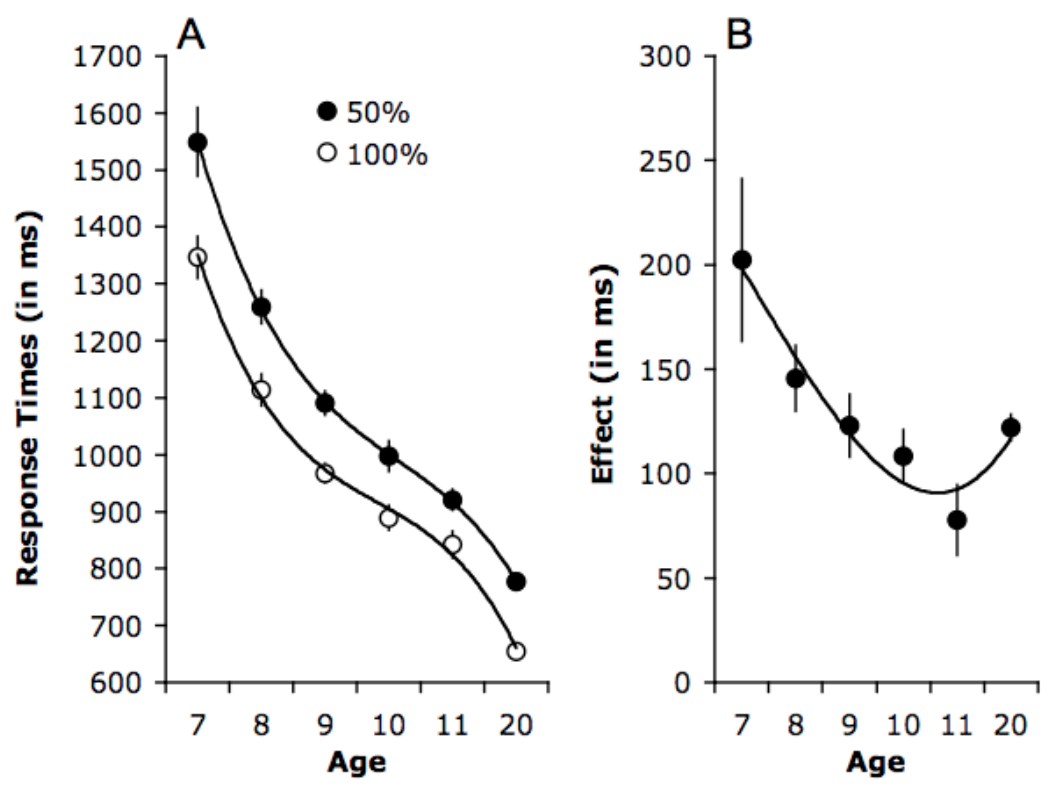

Figure 7. Between-experiment analysis of target conditions: salient item $50 \%$ relevant (Exp. 1) vs. salient item 100\% relevant (Exp. 2). (A) Mean RT and 1 SEM as a function of age and experimental context. (B) Mean and 1 SEM effect of knowledge about the probability that the salient item be the target (Exp.1 minus Exp. 2). 


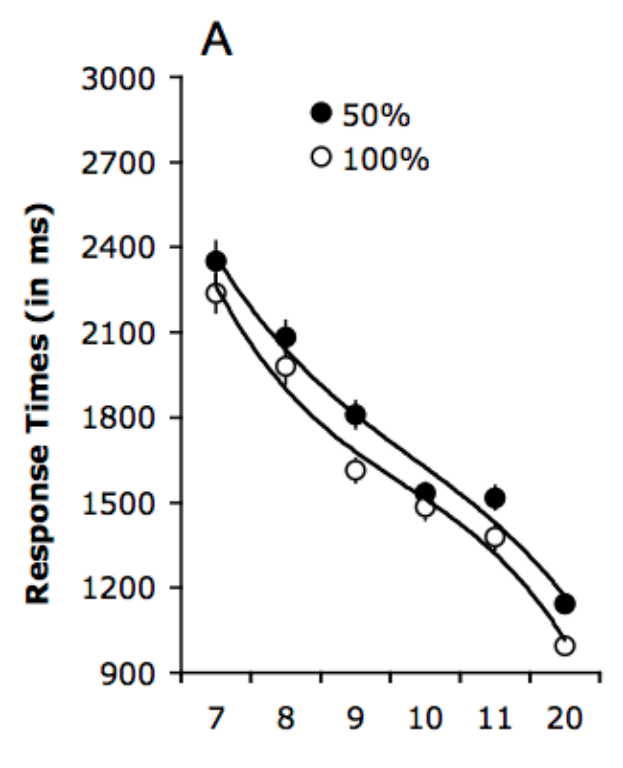

Age

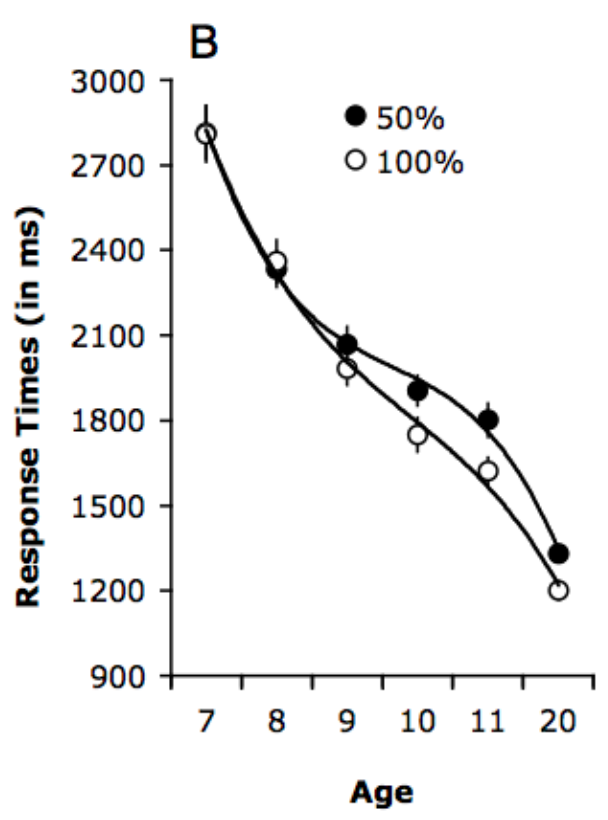

Figure 8. Between-experiment analysis of distractor conditions: salient item $50 \%$ irrelevant (Exp. 1) vs. salient item 100\% irrelevant (Exp. 3). (A) Mean RT and 1 SEM as a function of age and experimental context for 6-item displays. (B) Mean RT and 1 SEM as a function of age and experimental context for 10-item displays. 